\title{
Evolution towards the critical limit and the origin of Be stars
}

\author{
S. Ekström, G. Meynet, A. Maeder, and F. Barblan
}

Geneva Observatory, University of Geneva, Maillettes 51, 1290 Sauverny, Switzerland

e-mail: Sylvia.Ekstrom@obs.unige.ch

Received 15 June 2007 / Accepted 16 October 2007

\section{ABSTRACT}

\begin{abstract}
Context. More and more evidence leads to considering classical Be stars as rotating close to the critical velocity. If so, then the question that arises is the origin of this high surface velocity.

Aims. We determine which mechanisms accelerate the surface of single stars during the main sequence evolution. We study their dependence on the metallicity and derive the frequency of stars with different surface velocities in clusters of various ages and metallicities.

Methods. We have computed 112 stellar models of four different initial masses between 3 and $60 M_{\odot}$, at four different metallicities between 0 and 0.020 , and with seven different values of the ratio $\Omega / \Omega_{\text {crit }}$ between 0.1 and 0.99 . For all the models, computations were performed until either the end of the main sequence evolution or until the critical limit was reached.

Results. The evolution of surface velocities during the main sequence lifetime results from an interplay between meridional circulation (bringing angular momentum to the surface) and mass loss by stellar winds (removing it). The dependence on metallicity of these two mechanisms plays a key role in determining, for each metallicity, a limiting range of initial masses (spectral types) for stars able to reach or at least approach the critical limit. Present models predict a higher frequency of fast rotating stars in clusters with ages between 10 and 25 Myr. This is the range of ages where most of Be stars are observed. To reproduce the observed frequencies of Be stars, it is necessary to first assume that the Be star phenomenon already occurs for stars with $v / v_{\text {crit }} \geq 0.7$ and, second, that the fraction of fast rotators on the zero-age main sequence is higher at lower metallicities. Depending on the stage at which the star becomes a Be star, it may present either larger or less enrichments in nitrogen at the surface.
\end{abstract}

Key words. stars: evolution - stars: rotation - stars: emission-line, Be

\section{Introduction}

The aim of the present work is to study how the surface velocity varies as a function of time during the main sequence (MS) evolution for stars of various initial masses, metallicities, and rotation. Although predictions of the evolution of the surface velocity can already be found in the literature (see Heger \& Langer 2000; Meynet \& Maeder 2000, 2005), the published results lack homogeneity in their physical ingredients and cover ranges of initial velocities that are too narrow to provide valuable answers to the following questions:

- When is very fast rotation encountered in the course of the evolution of massive single stars in the MS phase?

- What is the percentage of stars rotating faster than a given value of $v_{\text {eq }} / v_{\text {crit }}$ in stellar clusters of various ages and metallicities?

- What are the expected chemical enrichments at the surface of these very fast rotating stars?

- What are the initial conditions required for single stars to reach the critical velocity limit (defined as the rotational velocity such that the centrifugal acceleration balances the gravity at the equator exactly)?

- Do these conditions depend on the mass and metallicity, and if so, how?

To know which stars can encounter the critical limit during the MS evolution is interesting in at least two astrophysical contexts: first, the study of Be stars; second, the very metal-poor stars representative of the stellar generations in the early Universe. Let us briefly describe the link with these two research areas.

\subsection{Be stars and the critical limit}

It is now widely accepted that the "Be phenomenon" is linked to fast rotation. Indeed, classical Be stars are B-type stars that exhibit line emission over the photospheric spectrum. This emission probably arises from an outwardly moving equatorial disk (Porter \& Rivinius 2003) whose formation is (at least in part) due to the fast rotation of the star. If the star is rotating with $v_{\text {eq }} / v_{\text {crit }} \sim 0.95$, the additional velocity needed to launch material into orbit is close to the speed of sound in the outer layers (Townsend et al. 2004). Therefore many instabilities, such as non-radial pulsations, begin to be effective for orbital ejection (Owocki 2004). Very interesting is that recent interferometric observations strongly support the idea that these stars are very fast rotators. The oblateness of the Be star Achernar (Domiciano de Souza et al. 2003) indeed suggests that the star is rotating near the critical limit, and Meilland et al. (2007) have for the first time obtained direct evidence that the disk around the Be star $\alpha$ Arae is in Keplerian rotation. The wind geometry is compatible with a thin disk wind and a polar-enhanced wind. These authors also find that $\alpha$ Arae is rotating very close to its critical rotation. Even the mystery behind the $\beta$ Ceph case seems to be elucidated: $\beta$ Ceph is a known Be star but also a known slow rotator, thus challenging the fast rotation scenario. However, $\beta$ Ceph is a multiple system. Schnerr et al. (2006) have shown that $\beta$ Ceph, the slow rotator, produces most of the light but is not responsible for the emission lines. The emission lines emanate from a much fainter companion, the Be star, which they consider as probably fast rotating. 
If the above observations do support the view that Be stars are at the critical limit, there are still some doubts that all Be stars are indeed at this limit. For instance, on the basis of the observed distribution of the $v \sin i$ for Be stars, Cranmer (2005) concludes that, while late type Be stars could be at the critical limit, early Be type stars might rotate at only $40 \%$ of their critical velocity. Let us note that the interpretation of the observations is stained with some theoretical uncertainties. A good example is the way the deduced velocities are affected because most of the radiative flux emanates from the polar regions for a fast rotating star (von Zeipel 1924). The low-velocity regions near the pole will thus have a stronger impact on the spectrum than the dimmer faster regions near the equator. This implies that the rotational velocity of very fast rotators has probably been systematically underestimated (Collins 2004; Townsend et al. 2004). Cranmer (2005) accounts for this effect in his work, but the problem is complex enough that the results are uncertain. Whether at the critical limit or not, Be stars represent wonderful laboratories for studying the effects of extreme rotation.

\subsection{Very metal poor stars and the critical limit}

The link between metal-free or very metal-poor stars and the question of the evolution of the surface velocity certainly deserves a lot of attention, since it might deeply affect the evolution of these stars. Let us recall that the radiation-driven stellar winds at low metallicity are much weaker than at high metallicity, thus it is commonly accepted that mass loss for very metal-poor stars is very inefficient. This might not be true, however, when rotation is taken into account in the models for at least two reasons (see Meynet et al. 2006b, to get a more detailed discussion of these points). First and somewhat paradoxically, since the stars are losing a very little amount of mass by radiatively driven stellar winds, they have more chance losing a large amount of mass by rotational ejection during the MS evolution. Indeed, because of the low line-driven winds, they will not be able to lose their angular momentum. They are thus more prone to reach the critical limit during their evolution (Maeder \& Meynet 2001; Meynet et al. 2006a). At the critical limit, as in the Be case, stellar matter is launched into an equatorial disk that will eventually dissipate by radiative effects. In that case, the material will be lost by the star. If correct, such an evolution would thus promote mass loss through a mechanical wind triggered by rotation. This "mechanical wind", as stressed above, appears to be more significant in metal-poor regions where the radiation-driven stellar winds are expected to be very low. Second, rotational mixing enhances the surface CNO content, which will lead to some increase in the outer layers opacities. This may revive the line-driven winds and lead to strong mass loss.

Such mass loss, either triggered by rotational ejection or through the enhancement of the CNO content of the outer layers, has (if realised) significant consequences on the evolution of the first stellar generations, as well as on their nucleosynthesis (Ekström et al. 2006; Hirschi 2007; Decressin et al. 2007). Also such mass loss may be significant in the process leading to the formation of a collapsar, which is the most favoured model for the progenitors of the long gamma ray bursts (Woosley 1993; Yoon \& Langer 2005; Woosley \& Heger 2006; Meynet \& Maeder 2007).

In the present work, we use rotating stellar models to address the questions of

- The possible origin of Be stars from a purely theoretical point of view: can models reproduce the way the number of
Be stars varies with the metallicity or with the age? Is their number high enough to account for the observed number of $\mathrm{Be}$ stars (supposing that Be stars are star rotating with values of $v_{\text {eq }} / v_{\text {crit }}$ higher than a given value)?

- The behaviour of rotating stars at very low or zero metallicity: how does the lack of metals influence the evolution of the equatorial velocity?

Of course, the answers given to these questions will strongly depend on the stellar models used. However, we think that this is worth to be done, because the stellar models we used were computed with physical ingredients that made predictions consistent with many observational features of massive stars (see Meynet \& Maeder 2005, and the references therein on the series of papers by the Geneva group on rotating models), and thus it is interesting to explore what these models say about fast-rotating stars in more detail. For very fast rotation, the present models probably still need to be improved by incorporating additional physics (e.g. pulsation), and we hope that the results obtained here will guide us towards improvements in the future.

The paper is organised as follow. In Sect. 2 the physical ingredients of the models are presented. We also recall some basic relations from the Roche model useful for discussing some properties of our models on the ZAMS. In Sect. 3, we present various characteristics of our models on the ZAMS as a function of the initial mass, velocity, and metallicity. The evolution of the models on the MS is presented in Sect. 4. The question of the initial conditions required to reach a given value of $v_{\mathrm{eq}} / v_{\text {crit }}$ during the MS phase is the subject of Sect. 5. The frequency of stars with velocities exceeding a given value of $v_{\text {eq }} / v_{\text {crit }}$ is presented in Sect. 6. The sensitivity of the predicted frequencies to various hypotheses made in this work are discussed in Sect. 7. Possible links with observational features of Be stars will be presented in Sect. 8. Conclusions and future perspectives are the subject of Sect. 9.

Let us mention that electronic tables are available for all our ZAMS and evolutionary models at http://obswww.unige. ch/Recherche/evol/Critical-limit-and-Be-stars

\section{Physics of the models}

\subsection{Input physics}

Since the detailed description of the physics of the models has already been presented in detail in previous papers (see references below), we shall keep the discussion very short here. The physics of rotation (transport of the angular momentum and of the chemical species) are treated as in Meynet \& Maeder (2005). These models are based on the theory of the transport mechanisms induced by rotation proposed by Zahn (1992) and further complemented by the works of Talon \& Zahn (1997) and Maeder \& Zahn (1998).

We computed the evolution of five different masses, $M=1$, $3,9,20,60 M_{\odot}$, at four different metallicities, $Z=0.020,0.002$, $0.00001,0$. For each combination of $M-Z$, we computed models with seven different initial rotational rates, $\omega=\Omega / \Omega_{\text {crit }}=0.10$, $0.30,0.50,0.70,0.80,0.90,0.99$. The 3 to $60 M_{\odot}$ models were evolved until either the beginning of core helium burning or reaching the break-up limit. For the $1 M_{\odot}$ models, only the ZAMS models are presented.

The initial composition of the models for the metallicities $Z=0.020,0.002,0.00001$, and 0 are respectively $X=0.7050$, $0.7545,0.7599$, and 0.76 and $Y=0.275,0.2435,0.24002$, and 0.24 . For the heavy elements, we used the same mixture 
as the one used in the OPAL opacity tables: the solar mixture of Grevesse \& Noels (1993) for $Z=0.020$ and 0.002 and the $\alpha$-enhanced mixture of Weiss (1995) for $Z=0.00001^{1}$. The nuclear reaction rates are from the NACRE database ${ }^{2}$.

The convection was treated according to the Schwarzschild criterion. The size of the convective core is increased by a moderate overshooting of $0.1 H_{\mathrm{p}}$.

The $3 M_{\odot}$ models were computed without mass loss, and the $9 M_{\odot}$ models were computed with the de Jager et al. (1988) prescription. For the higher mass models we used the prescription proposed by Kudritzki \& Puls (2000, hereafter KP00) and de Jager et al. (1988) in the temperature domain not covered by the first prescription. The effects of rotation on the mass loss rates are taken into account as explained in Maeder \& Meynet (2000a).

\subsection{The Roche model}

In all the derivations, we use the Roche model; i.e. we approximate the gravitational potential by $G M_{r} / r$ where $M_{r}$ is the mass inside the equipotential with a mean radius $r$. The radius $r$ that labels each equipotential is defined by $r=\left(V_{r} /(4 / 3 \pi)\right)^{1 / 3}$ where $V_{r}$ is the volume (deformed by rotation) inside the equipotential considered $^{3}$.

In the frame of the Roche model and for a conservative rotation law (i.e. such that the centrifugal force can be derived from a potential), the shape of a meridian at the surface of an equipotential is given by couples of $r$ and $\theta$ values satisfying the following equation:

$\frac{G M_{r}}{r}+\frac{1}{2} \Omega_{r}^{2} r^{2} \sin ^{2} \theta=\frac{G M_{r}}{r_{\mathrm{p}}}$,

where $r$ is the radius at colatitude $\theta, \Omega_{r}$ the angular velocity at that radius, $M_{r}$ the mass inside the considered equipotential surface, and $r_{\mathrm{p}}$ the radius at $\theta=0$. Thus the shape of a given equipotential is determined by three parameters $M_{r}, \Omega_{r}$, and $r_{\mathrm{p}}$. The first two, $M_{r}$, and $\Omega_{r}$ are independent variables. The third one is derived from the first two and the equations of stellar structure. Setting $x=\left(\frac{G M_{r}}{\Omega_{r}^{2}}\right)^{-1 / 3} r$, one can write Eq. (1) (see Kippenhahn $\&$ Thomas 1970)

$\frac{1}{x}+\frac{1}{2} x^{2} \sin ^{2} \theta=\frac{1}{x_{\mathrm{p}}}$

With this change of variable, the shape of an equipotential is uniquely determined by only one parameter $x_{\mathrm{p}}$.

With $M$ the total mass of the star and $\Omega$ the angular velocity at the surface, Eqs. (1) or (2) give the shape of a meridian line at the stellar surface. Setting

$f=\frac{R_{\mathrm{e}}}{R_{\mathrm{p}}}$,

${ }^{1}$ See http://www-phys.1lnl.gov/Research/OPAL/opal. $\mathrm{html}$ for exact references.

${ }^{2}$ See http://pntpm.ulb.ac.be/Nacre/barre_database.htm

3 ZAMS models are supposed to rotate like solid bodies, the centrifugal force is conservative, and it is possible to define equipotential surfaces. This is no longer the case for shellular rotation. In that case, however, the shape of the isobaric surfaces are given by the same expression as the one giving the shape of the equipotentials for solid body rotation provided some changes in variables are performed (see Meynet \& Maeder 1997). where $R_{\mathrm{e}}$ is the equatorial radius, one easily obtains from Eq. (1) that

$R_{\mathrm{p}}=\left(\frac{G M}{\Omega^{2}}\right)^{1 / 3}\left(\frac{2(f-1)}{f^{3}}\right)^{1 / 3}=\left(\frac{G M}{\Omega^{2}}\right)^{1 / 3} x_{\mathrm{p}}$

The above equation relates the inverse of the oblateness $f$ to $R_{\mathrm{p}}$.

As recalled above, the critical angular velocity, $\Omega_{\text {crit }}$, corresponds to the angular velocity at the equator of the star such that the centrifugal force balances the gravity exactly. The classical critical angular velocity or the $\Omega$-limit (to distinguish it from the $\Omega \Gamma$-limit as defined in Maeder \& Meynet 2000a) in the frame of the Roche model is given by

$\Omega_{\text {crit }}=\left(\frac{2}{3}\right)^{\frac{3}{2}}\left(\frac{G M}{R_{\mathrm{p}, \text { crit }}^{3}}\right)^{\frac{1}{2}}$,

where $R_{\mathrm{p}, \text { crit }}$ is the polar radius when the surface rotates with the critical velocity. The critical velocity is given by

$v_{\text {crit }}=\left(\frac{2}{3} \frac{G M}{R_{\mathrm{p}, \text { crit }}}\right)^{\frac{1}{2}}$.

By replacing $\Omega$ in Eq. (4) by $\left(\Omega / \Omega_{\text {crit }}\right) \cdot \Omega_{\text {crit }}$ and using Eq. (5), one obtains a relation between $R_{\mathrm{p}}, f$, and $R_{\mathrm{p}, \text { crit }}$,

$R_{\mathrm{p}}=\frac{3}{2} R_{\mathrm{p}, \mathrm{crit}}\left(\frac{\Omega_{\mathrm{crit}}}{\Omega}\right)^{2 / 3}\left(\frac{2(f-1)}{f^{3}}\right)^{1 / 3}$.

Thus one has

$\frac{\Omega}{\Omega_{\text {crit }}}=\left(\frac{3}{2}\right)^{3 / 2}\left(\frac{R_{\mathrm{p}, \text { crit }}}{R_{\mathrm{p}}}\right)^{3 / 2}\left(\frac{2(f-1)}{f^{3}}\right)^{1 / 2}$.

With a good approximation (see below), one finds that $R_{\mathrm{p}, \text { crit }} / R_{\mathrm{p}} \simeq 1$ and therefore

$\frac{\Omega}{\Omega_{\text {crit }}} \simeq\left(\frac{3}{2}\right)^{3 / 2}\left(\frac{2(f-1)}{f^{3}}\right)^{1 / 2}$.

This equation is quite useful since it allows determination of $\Omega_{\text {crit }}$ from quantities obtained with a model computed for any $\Omega<$ $\Omega_{\text {crit }}$; however, the closer to the critical value, the better. This is not the case of Eq. (7) which involves $R_{\mathrm{p}, \text { crit }}$ whose knowledge can only be obtained by computing models at the critical limit. Below, we see to what extent the use of expression (9) provides a good approximation of $\Omega_{\text {crit }}$.

Setting $v$ the velocity at the equator, one finds that

$\frac{v}{v_{\text {crit }}}=\frac{\Omega R_{\mathrm{e}}}{\Omega_{\mathrm{c}} R_{\mathrm{e}, \text { crit }}}=\frac{\Omega}{\Omega_{\text {crit }}} \frac{R_{\mathrm{e}}}{R_{\mathrm{p}}} \frac{R_{\mathrm{p}}}{R_{\mathrm{p}, \text { crit }}} \frac{R_{\mathrm{p}, \text { crit }}}{R_{\mathrm{e}, \text { crit }}}$,

where $R_{\mathrm{e}, \text { crit }}$ is the equatorial radius when the surface rotates with the critical velocity. Using Eq. (7) above, and the fact that in the Roche model $R_{\mathrm{p}, \text { crit }} / R_{\mathrm{e} \text {,crit }}=2 / 3$, one obtains

$\frac{v}{v_{\text {crit }}}=\left(\frac{\Omega}{\Omega_{\text {crit }}} 2(f-1)\right)^{1 / 3}$.

An interesting quantity is the ratio of the centrifugal acceleration $a_{\text {cen }}$ to the gravity $g_{\mathrm{e}}$ at the equator

$\frac{a_{\text {cen }}}{g_{\mathrm{e}}}=\frac{\Omega^{2} R_{\mathrm{e}}^{3}}{G M}=\left(\frac{\Omega}{\Omega_{\text {crit }}}\right)^{2}\left(\frac{2}{3}\right)^{3} f^{3}\left(\frac{R_{\mathrm{p}}}{R_{\mathrm{p}, \text { crit }}}\right)^{3}$, 


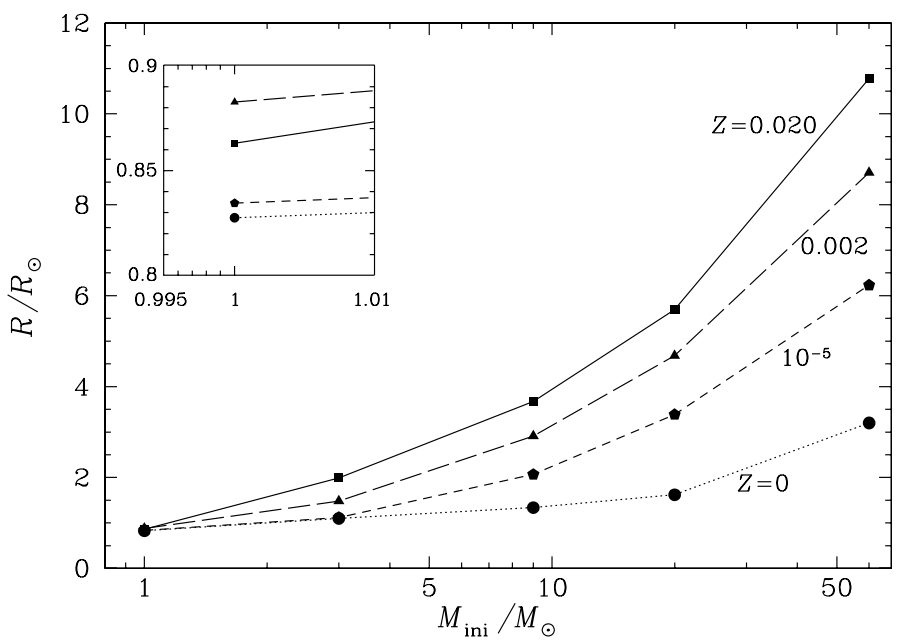

Fig. 1. Variations in the radius as a function of initial mass, for various metallicities for non-rotating stars. The inset zoom shows the small difference in radii in the case of the $1 M_{\odot}$ models.

where we use Eq. (5) and divide/multiply by $R_{\mathrm{p}, \text { crit }}^{3}$. Replacing $R_{\mathrm{p}, \text { crit }} / R_{\mathrm{p}}$ by its expression deduced from Eq. (7), we obtain

$\frac{a_{\text {cen }}}{g_{\mathrm{e}}}=2(f-1)$.

We can check that at the critical limit, when $f=3 / 2$, then $a_{\text {cen }}=g_{\mathrm{e}}$.

\section{Basic parameters on the zero age main sequence}

We consider that a star has arrived on the ZAMS, when a mass fraction of 0.003 of hydrogen has been transformed into helium at the centre. At this stage, the star is supposed to have a solid body rotation. We present here the stellar radii, the total luminosity, the variation with the colatitude of the effective temperature, and the moment of inertia of the models. We then show the relations between the initial rotation and the angular momentum content, $v_{\text {ini }} / v_{\text {crit }}$ and $\Omega_{\text {ini }} / \Omega_{\text {crit }}$.

\subsection{Radii, luminosities, and effective temperatures}

Considering first the case without rotation, we can see in Fig. 1 that there is a relation between radius and mass of the form $R \sim M^{0.6}$ at standard metallicity. This relation becomes shallower with decreasing $Z$, down to $R \sim M^{0.3}$ at $Z=0$. This comes mainly from two causes: 1) first, at lower metallicity, the opacities are lower; 2) the CNO elements are less abundant, or even absent in metal-free stars. To compensate for the energy radiated by the surface when smaller amounts of $\mathrm{CNO}$ elements are present, the central regions must extract energy from nuclear reactions at a higher temperature regime. To reach this regime, the star contracts more during the pre-MS phase and is thus more compact on the ZAMS. For example, in the case of the $60 M_{\odot}$ models, the radius at $Z=0$ is reduced by a factor 3.36 in comparison with the radius at standard metallicity. The $1 M_{\odot}$ models are running only on $p p$-chains and show very small differences in their radii: only about $5 \%$ between $Z=0.020$ and $Z=0$ (see the inset zoom in Fig. 1).

In Fig. 2, we present the variation in the polar radius $R_{\mathrm{p}}$ as a function of $\omega=\Omega / \Omega_{\text {crit }}$, normalized to its non-rotating value, for the standard metallicity models. We see that increasing $\omega$

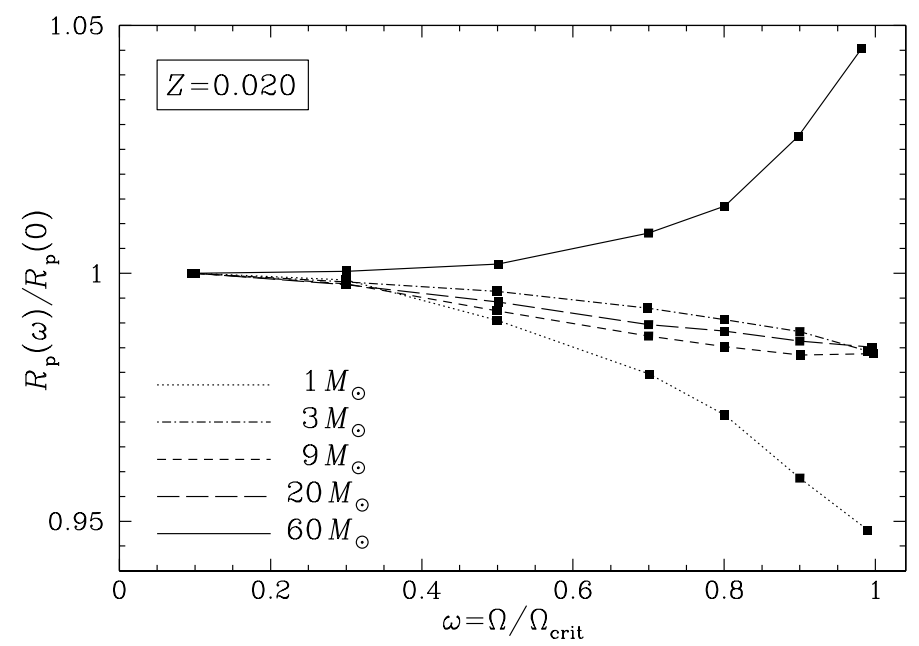

Fig. 2. Variations in the polar radius as a function of the ratio $\omega=$ $\Omega / \Omega_{\text {crit }}$, normalized to the non-rotating value, for various masses at standard metallicity.

tends to decrease the radius in the case of the 1 to $20 M_{\odot}$, a trend that can be explained as follows: rotation plays the role of a sustaining force in the star. Thus, the central temperature and density in rotating stars are lower, subsequently decreasing the luminosity and the radius. The change of the polar radius for the $1 M_{\odot}$ model amounts to about $5 \%$. In the case of the 3,9 , and $20 M_{\odot}$ models, the assumption of $R_{\mathrm{p}, \text { crit }} / R_{\mathrm{p}}=1$ (Sect. 2.2) is absolutely valid, since the decrease only amounts to $1.5 \%$. Why are the nearly completely radiative stars such as the $1 M_{\odot}$ stellar models more deformed for a given value of $\omega$ than stars with a significant convective core? It comes from the fact that the density gradient in convective zones is shallower than in radiative ones, allowing more mass to be contained in a given volume. This strengthens gravity and the resistance to deformation.

For the $60 M_{\odot}$ models, the change in the polar radius has the same amplitude as for the $1 M_{\odot}$ model but in the opposite direction, showing an increase of about $4.5 \%$. How such a difference of behaviour between the lowest and highest mass models might be explained? In the high-mass star range, radiation pressure contributes more to the total pressure. It is the highest in the polar regions where the effective gravity (gravity decreased by the centrifugal acceleration) is the highest (von Zeipel theorem). A look at Fig. 6 shows that $T_{\text {eff }}$ (pole) is higher when $\omega$ is higher (10\% between $\omega=0.1$ and $\omega=0.99$ ), and therefore $P_{\text {rad }} \sim T^{4}$ is stronger. This tends to inflate the polar radius in massive stars.

Rotation deforms the star, the ratio $R_{\mathrm{e}} / R_{\mathrm{p}}$ increasing with $\omega$ as shown in Fig. 3. We have plotted the simplified relation obtained by Eq. (9), assuming $R_{\mathrm{p}, \text { crit }} / R_{\mathrm{p}}=1$ (continuous line), as well as the relation obtained using Eq. (8) applied to two different masses ( 1 and $60 M_{\odot}$ ) at $Z=0.02$. In this case, we observe the direct effect of the behaviour seen in Fig. 2 . For the $1 M_{\odot}$, the decrease in $R_{\mathrm{p}}$ with increasing $\omega$ makes $f$ higher than the continuous line, joining it only at the critical limit since $R_{\mathrm{e}, \text { crit }} / R_{\mathrm{p}, \text { crit }}$ is always equal to 1.5 in the Roche model frame, while the inverse behaviour of the $60 M_{\odot}$ leads to the inverse result. Let us note also that in all cases, the equatorial radius becomes longer than 1.1 times the polar one only for $\omega \geq 0.75$.

In Fig. 4, we show the behaviour of the luminosity $L(\omega)$ of our $60 M_{\odot}$ models as a function of $\omega$ for the different metallicities considered. The value of the luminosity is normalized to its non-rotating value. One sees that the luminosity decreases by at most 5 to $10 \%$ when rotation increases up to the critical 


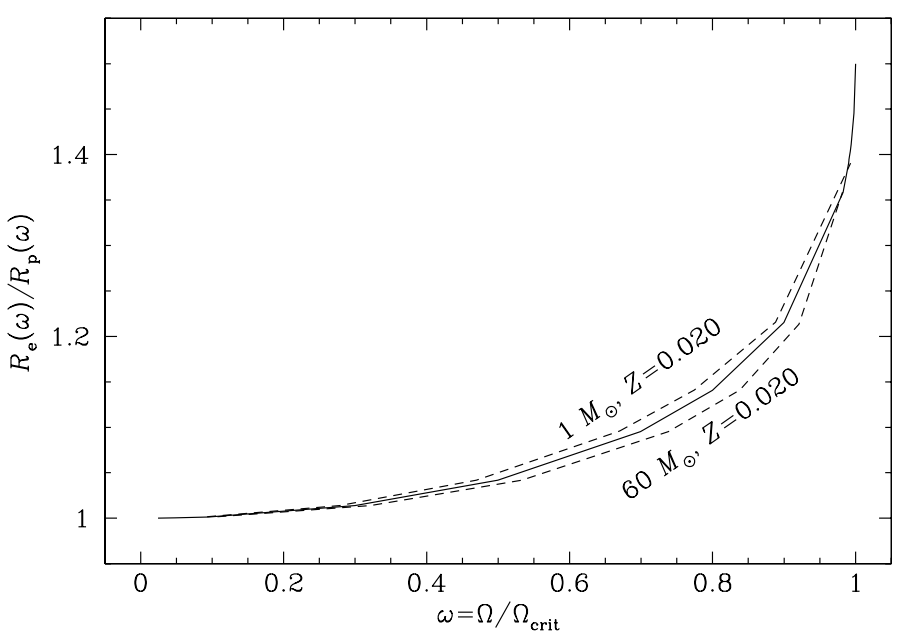

Fig. 3. Variation in the ratio $R_{\mathrm{e}}(\omega) / R_{\mathrm{p}}(\omega)$ (equatorial over polar radius) as a function of $\omega=\Omega / \Omega_{\mathrm{c}}$. The continuous line shows the relation given by Eq. (9), assuming $R_{\mathrm{p}, \mathrm{crit}} / R_{\mathrm{p}}=1$. The dashed lines show the relations for the $Z=0.020$ models with 1 and $60 M_{\odot}$ using Eq. (8).

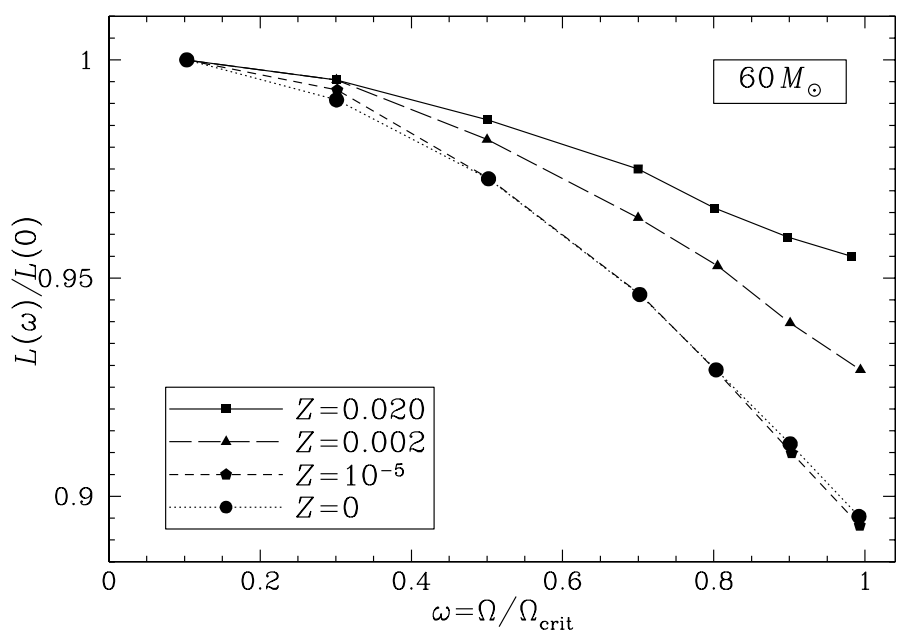

Fig. 4. Variations in the total luminosity as a function of $\omega$, normalized to the non-rotating value, for $60 M_{\odot}$ at various metallicities.

limit. As mentioned above, this is because the centrifugal acceleration helps in sustaining the star against the gravity and allows the luminosity to take a value corresponding to a non-rotating lower initial mass star. We can see that the effects of rotation are stronger at lower metallicity. This is related to the fact that low metallicity stars are more compact. The regions having a temperature high enough to undergo nuclear burning are more extended in the star and thus overlap regions that are affected more by rotation.

Figure 5 is the same as Fig. 4, but this time by fixing the metallicity $(Z=0.020)$ and varying the mass. The $1 M_{\odot}$ is the most affected by rotation, with a decrease of $22.5 \%$ in its luminosity at break-up. The higher masses only show a decrease of 4.5 to $7 \%$. This again is related to the energy production through $p p$-chains in the $1 M_{\odot}$ model being more extended in the interior of the star. As above, a non-negligible part of the luminosity is produced in regions that may suffer from the effects of rotation.

Figure 6 shows the variation with colatitude in the effective temperature at the surface of a $20 M_{\odot}$ star for various rotation rates at standard metallicity. The ratio $\left(T_{\mathrm{eff}}(\right.$ pole)$T_{\text {eff }}$ (equator)) $/ T_{\text {eff }}$ (equator) becomes superior to $10 \%$ only for

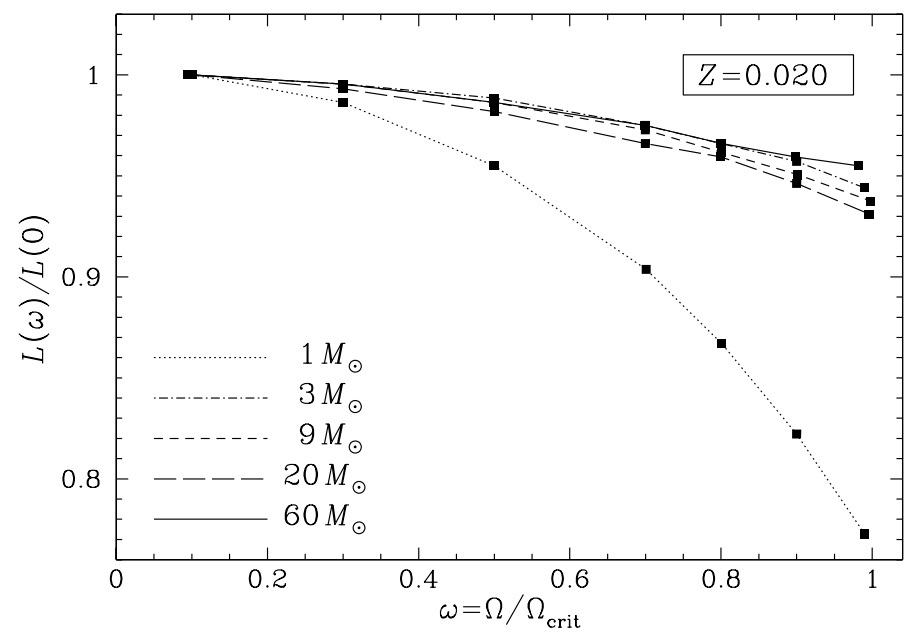

Fig. 5. Variations in the total luminosity as a function of $\omega$, normalized to the non-rotating value, for various masses at standard metallicity.

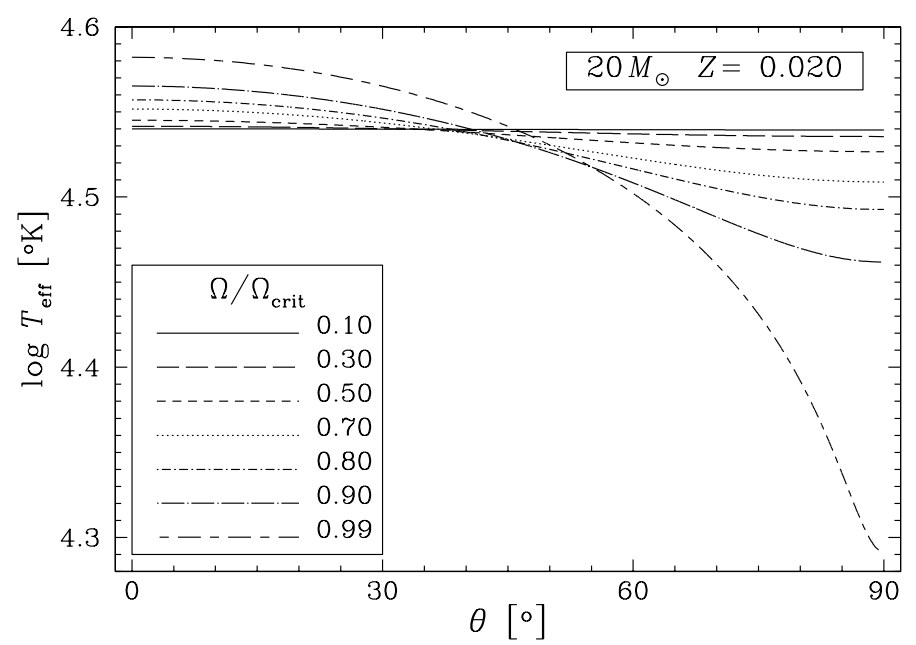

Fig. 6. Variations in the effective temperature $T_{\text {eff }}$ as a function of colatitudinal angle $\theta$, for the various values of rotational rates, in the $20 M_{\odot}$ model at standard metallicity.

$\omega>0.7$. Near break-up, the effective temperature of the polar region is about a factor two higher than that of the equatorial one. We may compare this result with the recent work of Espinosa Lara \& Rieutord (2007) who have studied the structure and dynamics of rapidly rotating stellar models with a two-dimension code. They computed a model with $\omega=0.82$ for which the ratio ( $T_{\text {eff }}$ (pole) $-T_{\text {eff }}$ (equator) $) / T_{\text {eff }}$ (equator) is $14 \%$. Our closer model $(\omega=0.80)$ shows a slightly higher ratio of $16 \%$, though we should expect a lower value due to the lower $\omega$. Let us note that the 2D simulation has been carried out within a spherical box, which according to the authors, may soften the latitudinal variations. It would be interesting to check how much of the discrepancy remains were the $2 \mathrm{D}$ computation done without such a container.

\subsection{Inertia, angular momenta, and rotational energies}

Figure 7 presents the variations in the moment of inertia with the initial mass of the models. These variations show almost no dependence on $\omega$, so only the case of $\omega=0.1$ is plotted. The moments of inertia have been obtained by summing the contributions of each shell $i$ of the model: $I=\sum_{i}(2 / 3) r_{i}^{2} \mathrm{~d} m_{i}$, where $r_{i}$ 


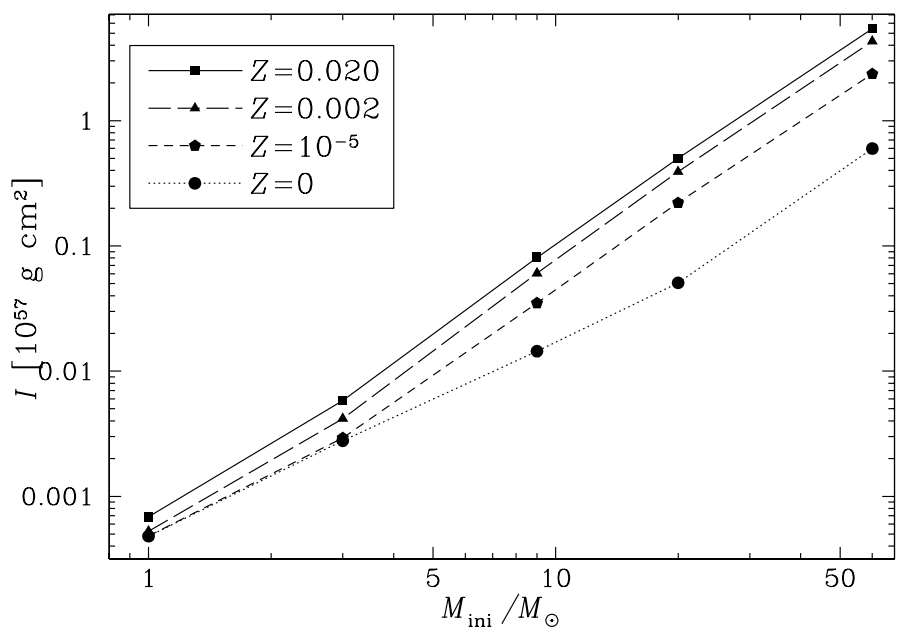

Fig. 7. Variations in the moment of inertia as a function of initial mass for various metallicities (models with $\Omega / \Omega_{\text {crit }}=0.1$ ).
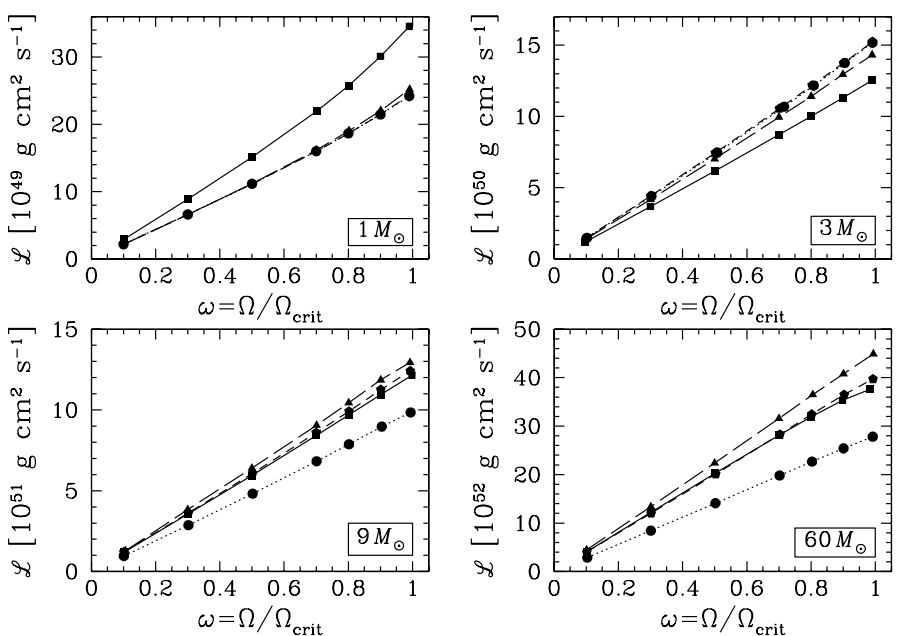

Fig. 8. Variations in the total angular momentum content of the models on the ZAMS as a function of initial rotation rate $\omega=\Omega / \Omega_{\text {crit }}$ for various metallicities, plotted according to the initial mass of the models. The coding for $Z$ is the same as in Fig. 7.

is the mean radius of the shell, i.e. the radius in $P_{2}(\cos \theta)=0$. Thus, $r_{i}$ accounts for the rotational distortion up to the secondorder Legendre polynomial, and the above way of calculating the moment of inertia is correct at this level of approximation. Let us mention that rotation deforms only the outermost layers of the model, which contain very little mass. This is why the moment of inertia does not change much with or without rotation.

We note that, at a given metallicity, the moment of inertia $I$ increases with the initial mass. The increase amounts to about 4 orders of magnitudes between 1 and $60 M_{\odot}$. As can be seen in Fig. 7, the relation between $I$ and $M$ is almost linear on a logarithmic scale, with a slope of 2.2 for the non-zero metallicities and of 1.7 for $Z=0$. The difference in the slopes can be linked to the difference in the way the radii increase with increasing mass. From the mass-radius relations obtained in Sect. $3.1\left(R \sim M^{0.6}\right.$ at standard metallicity and $R \sim M^{0.3}$ at $Z=0$ ), we indeed expect a relation $I \sim M^{2.2}$ and $I \sim M^{1.6}$ at $Z=0.02$ and 0 , respectively. For a given initial mass, the moments of inertia are smaller at lower metallicities, as expected due to smaller radii at lower $Z$.

The variation in the total angular momentum content $(\mathcal{L}=$ $\left.\sum_{i} I_{i} \Omega_{i}\right)$ as a function of $\omega$ is shown in Fig. 8. Note that the highest point on the right of the figures gives an estimate of the
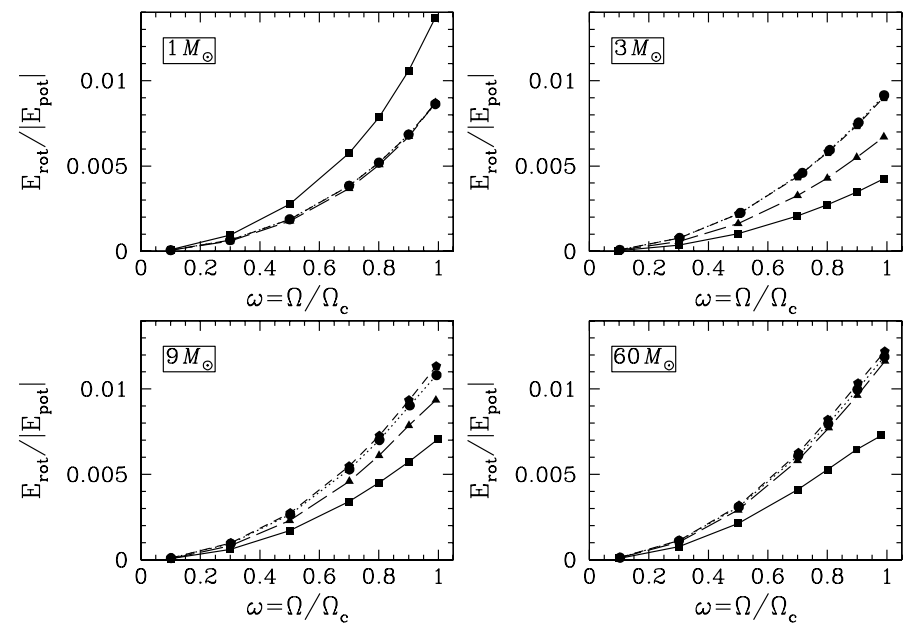

Fig. 9. Variations in the ratio between rotational and potential energy as a function of the ratio $\omega=\Omega / \Omega_{\text {crit }}$ for various metallicities, plotted according to the initial mass of the model. The coding for $Z$ is the same as in Fig. 7.

maximum angular momentum content on the ZAMS (for solid body rotation). Since the moment of inertia of the star does not depend much on the rotation velocity, and since we suppose solid body rotation on the ZAMS, one obtains quasi linear relations between $\mathcal{L}$ and $\omega$. We note that the metallicity dependence remains modest at least in the range of metallicities between 0.00001 and 0.020 . In the 9 to $60 M_{\odot}$ range, the value of $\omega$ being kept constant, there is at first a slight increase in the angular momentum content when decreasing the metallicity (compare the curve with the black squares for the $Z=0.020$ models with the curve with the triangles corresponding to 0.002 models). Then for still lower metallicities, the angular momentum decreases with the metallicity (the curve for the $Z=0.00001$ models overlaps the $Z=0.020$ models) reaching its lower values for the metal-free stellar models.

In the mass range between 9 and $20 M_{\odot}$, stars having the same angular momentum content on the ZAMS lead to a very similar value for $\omega$, whatever their metallicity between 0.00001 and 0.02 . Only if the metallicity is zero would the value of $\omega$ corresponding to the same angular momentum content be much higher. As a numerical example, a $20 M_{\odot}$ on the ZAMS with $\mathcal{L}=0.3 \times 10^{53} \mathrm{~cm}^{2} \mathrm{~g} \mathrm{~s}^{-1}$ has a ratio $\omega=0.5$ when $Z$ ranges between 0.00001 and 0.020 . This angular momentum content corresponds to $\omega=0.8$ when $Z=0$.

The rotational kinetic energy $\left(E_{\mathrm{rot}}=\sum_{i}(1 / 2) I_{i} \Omega_{i}^{2}\right)$ expressed as a fraction of the binding energy $\left(E_{\mid \text {pot } \mid}=\right.$ $\left.-G \sum_{i}\left(M_{i} / r_{i}\right) \mathrm{d} m_{i}\right)$ is shown in Fig. 9. The rotational energy amounts to at most one percent of the binding energy. This is consistent with the well known fact that the effects of the centrifugal acceleration remains quite modest on the hydrostatic structure of the stellar interior, even when the surface rotates with a velocity near the critical one. For a $60 M_{\odot}$ stellar model at $Z=0.020$, the binding energy is $1.8 \times 10^{51} \mathrm{erg}$, while its $Z=0$ counterpart has a binding energy equal to $5.3 \times 10^{51} \mathrm{erg}$, i.e. about a factor 3 higher. Let us recall for comparison that the binding energy of a neutron star is of the order of $10^{53} \mathrm{erg}$, about two orders of magnitude greater. 


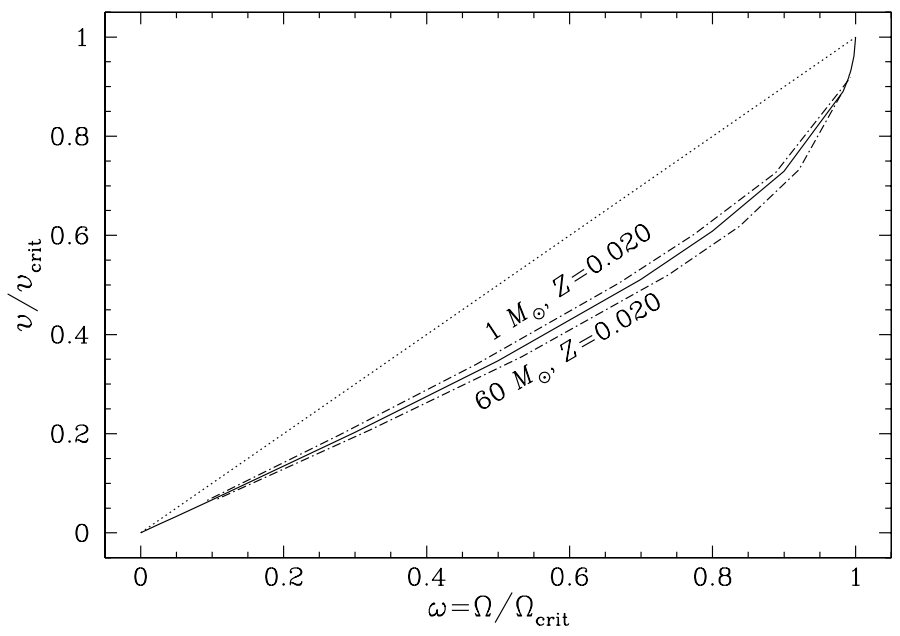

Fig. 10. Relation between $v / v_{\text {crit }}$ and $\omega=\Omega / \Omega_{\text {crit }}$ obtained in the frame of the Roche model. The continuous line is obtained assuming $R_{\mathrm{p}, \text { crit }} / R_{\mathrm{p}}=1$ (see text and Eqs. (9) and (11)). The dot-dashed lines show the relations for the $Z=0.020$ models with 1 and $60 M_{\odot}$ using Eq. (8). The dotted line is the line of slope 1.

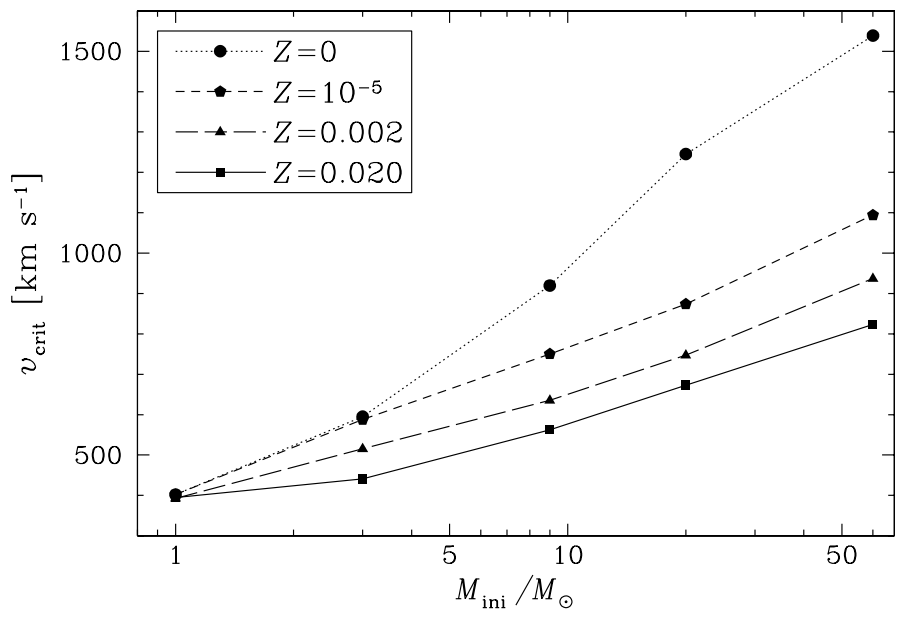

Fig. 11. Variations in the critical equatorial velocity on the ZAMS as a function of the initial mass, for various metallicities.

\subsection{Relations between $\Omega / \Omega_{\text {crit }}$ and $v / v_{\text {crit }}$}

The relations between $v / v_{\text {crit }}$ and $\Omega / \Omega_{\text {crit }}$ obtained in the frame of the Roche model (see Eq. (11)) for the 1 and $60 M_{\odot}$ stellar models at $Z=0.02$ are shown in Fig. 10. In case we suppose that the polar radius remains constant $\left(R_{\mathrm{p}, \text { crit }} / R_{\mathrm{p}}=1\right)$, then Eq. (9) can be used and one obtains a unique relation between $\Omega / \Omega_{\text {crit }}$ and $v / v_{\text {crit }}$, independent of the mass, metallicity, and evolutionary stage considered (continuous line). One sees that the values of $v / v_{\text {crit }}$ are lower than that of $\Omega / \Omega_{\text {crit }}$ by at most $\sim 25 \%$. At the two extremes, the ratios are of course equal.

\subsection{Velocities on the ZAMS: $v_{\text {crit }}, v$}

Table 1 gives the angular velocity, the period, the polar radius, and the equatorial velocity at the critical limit on the ZAMS for the various models. Let us note that, for obtaining the value of $\Omega_{\text {crit }}$ or of whatever quantities at the critical limit, it is necessary to compute models at the critical limit. Here we have computed models very near, but not exactly at, the critical limit, since at that point numerical singularities are encountered. As explained in Sect. 2.2, using Eq. (9) we could have estimated
Table 1. Angular velocity, rotational period, polar radius, and equatorial velocity at the critical limit on the ZAMS (solid body rotation).

\begin{tabular}{llrrrr}
\hline \hline$M$ & $Z$ & $\begin{array}{c}\Omega_{\text {crit }} \\
\mathrm{s}^{-1}\end{array}$ & $\begin{array}{r}P_{\text {crit }} \\
\mathrm{h}\end{array}$ & $\begin{array}{r}R_{\mathrm{p}, \text { crit }} \\
R_{\odot}\end{array}$ & $\begin{array}{r}v_{\text {crit }} \\
\mathrm{km} \mathrm{s}^{-1}\end{array}$ \\
\hline 1 & 0 & $4.9 \mathrm{E}-04$ & 3.6 & 0.8 & 400 \\
1 & $10^{-5}$ & $4.9 \mathrm{E}-04$ & 3.6 & 0.8 & 400 \\
1 & 0.002 & $4.6 \mathrm{E}-04$ & 3.8 & 0.8 & 390 \\
1 & 0.020 & $4.6 \mathrm{E}-04$ & 3.8 & 0.8 & 395 \\
& & & & & \\
3 & 0 & $5.3 \mathrm{E}-04$ & 3.3 & 1.1 & 595 \\
3 & $10^{-5}$ & $5.1 \mathrm{E}-04$ & 3.4 & 1.1 & 585 \\
3 & 0.002 & $3.4 \mathrm{E}-04$ & 5.1 & 1.4 & 515 \\
3 & 0.020 & $2.2 \mathrm{E}-04$ & 8.1 & 2.0 & 440 \\
& & & & & \\
9 & 0 & $6.5 \mathrm{E}-04$ & 2.7 & 1.4 & 920 \\
9 & $10^{-5}$ & $3.5 \mathrm{E}-04$ & 4.9 & 2.0 & 750 \\
9 & 0.002 & $2.2 \mathrm{E}-04$ & 8.1 & 2.8 & 635 \\
9 & 0.020 & $1.5 \mathrm{E}-04$ & 11.7 & 3.6 & 560 \\
& & & & & \\
20 & 0 & $7.3 \mathrm{E}-04$ & 2.4 & 1.6 & 1245 \\
20 & $10^{-5}$ & $2.5 \mathrm{E}-04$ & 7.0 & 3.3 & 870 \\
20 & 0.002 & $1.6 \mathrm{E}-04$ & 11.1 & 4.6 & 745 \\
20 & 0.020 & $1.2 \mathrm{E}-04$ & 15.2 & 5.6 & 670 \\
& & & & & \\
60 & 0 & $4.6 \mathrm{E}-04$ & 3.8 & 3.2 & 1540 \\
60 & $10^{-5}$ & $1.7 \mathrm{E}-04$ & 10.6 & 6.4 & 1095 \\
60 & 0.002 & $1.0 \mathrm{E}-04$ & 16.9 & 8.7 & 935 \\
60 & 0.020 & $7.0 \mathrm{E}-05$ & 24.9 & 11.3 & 820 \\
\hline
\end{tabular}
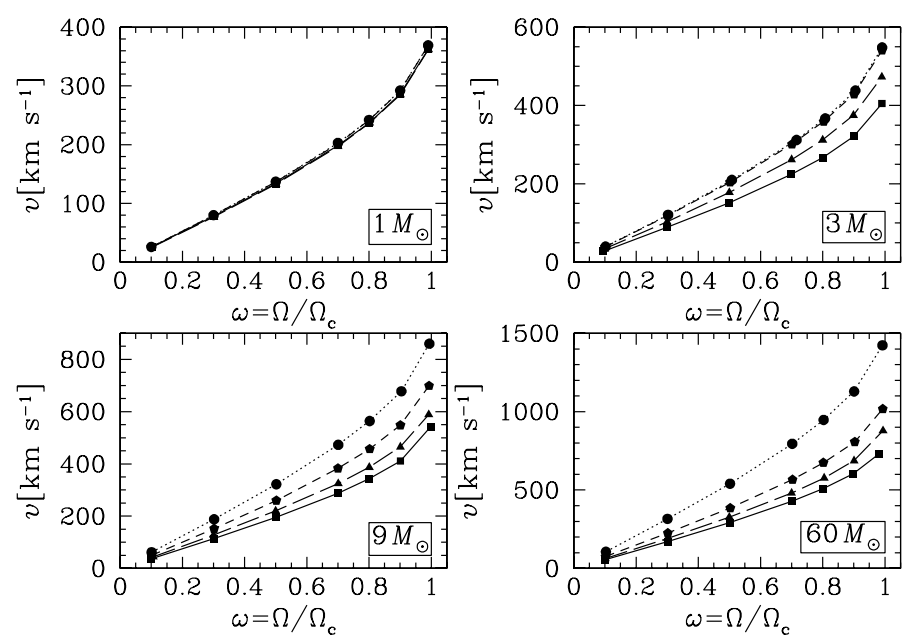

Fig. 12. Variations in the surface equatorial velocity on the ZAMS as a function of initial rotation rate $\omega=\Omega / \Omega_{\text {crit }}$ for various metallicities and masses. The coding for $Z$ is the same as in Fig. 11.

$\Omega_{\text {crit }}$ from whatever model was computed with a lower initial velocity. Doing this for the whole range of initial masses, metallicities and velocities, we would have obtained values within $10 \%$ of those shown in Table 1 . For the models between 3 and $20 M_{\odot}$, the error would always be inferior to $3.5 \%$ because in this mass range, $R_{\mathrm{p}}(\omega) / R_{\mathrm{p}}(0)$ remains very near 1 (see Fig. 2) and Eq. (9) is a good approximation of Eq. (8). For the 1 and $60 M_{\odot}$, the errors would be larger, amounting to a maximum of 9.3 and $6.7 \%$ respectively, showing the need to use instead Eq. (8) if $\omega \ll 1$. In general the error decreases drastically when $\omega$ increases, so to obtain the values given in Table 1, we have used our faster rotating model, deriving the value of $\Omega_{\text {crit }}$ (using Eq. (9)) with an error less than $0.001 \%$. The critical period, $P_{\text {crit }}$ is then simply given by $P_{\text {crit }}=2 \pi / \Omega_{\text {crit }}$. The polar radius is obtained 

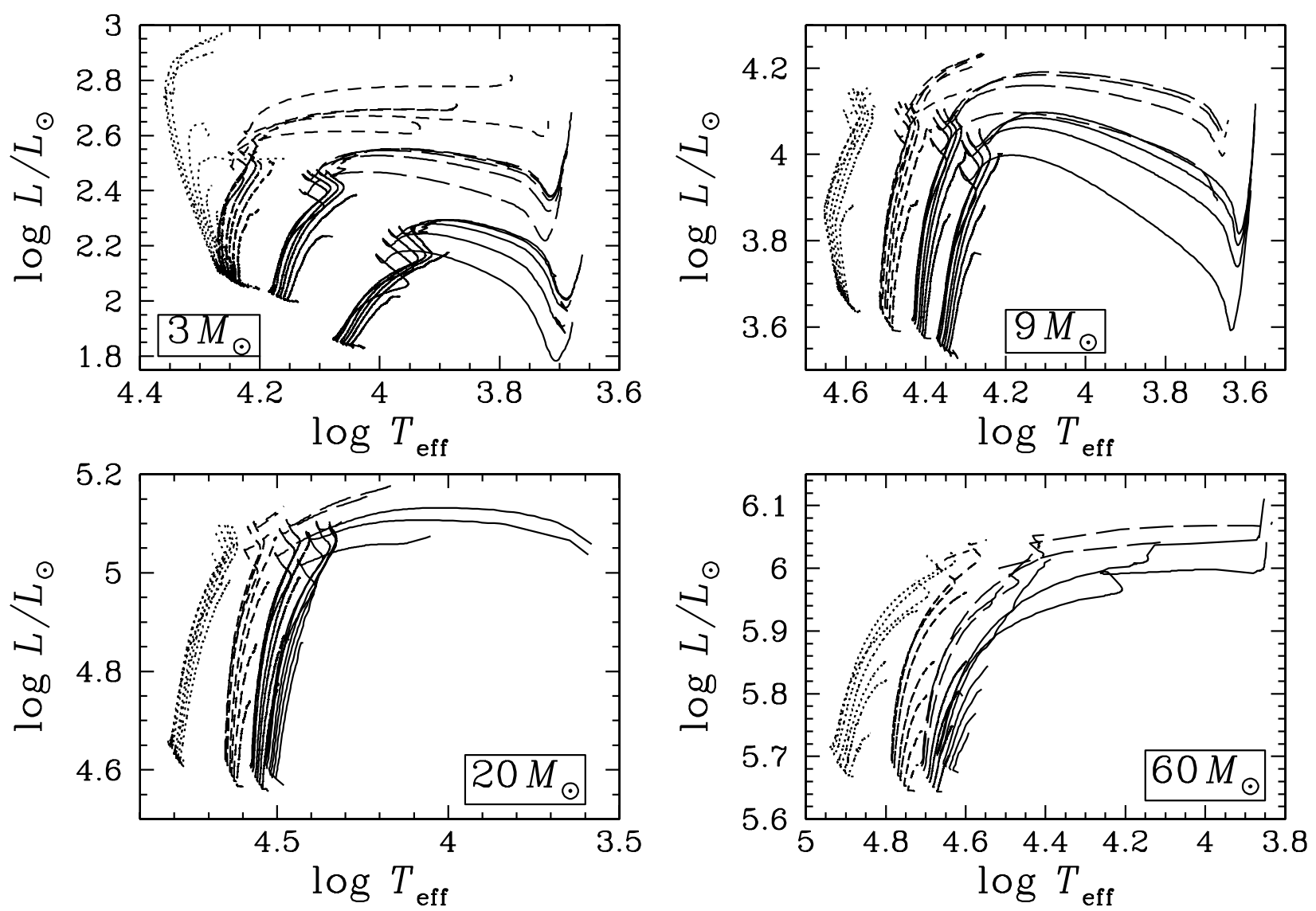

Fig. 13. Evolutionary tracks in the theoretical Hertzsprung-Russell diagrams for all the models computed beyond the ZAMS. The effective temperature corresponds to a surface-averaged effective temperature. The computations are stopped when the star reaches the critical limit or before the beginning of the core He-burning phase.

from Eq. (5) and the critical equatorial velocity is deduced from $v_{\text {crit }}=1.5 R_{\text {p,crit }} \Omega_{\text {crit }}$.

The variation in the critical velocity as a function of the initial mass and metallicity is shown in Fig. 11. For a given initial mass, the critical velocity is higher at lower $Z$ : e.g. for the $60 M_{\odot}$ model, the critical velocity goes from slightly more than $800 \mathrm{~km} \mathrm{~s}^{-1}$ at $Z=0.020$ to more than $1500 \mathrm{~km} \mathrm{~s}^{-1}$ at $Z=0$. It is a consequence of the smaller radii of stars at low metallicity (see Eq. (6)) as can be seen from Fig. 1.

At a given metallicity, the critical velocity becomes higher for higher initial masses. This reflects the fact that the ratio of the mass to the radius increases with the star mass (cf. Sect. 3.1). The critical rotation periods are between 2.4 and $25 \mathrm{~h}$ for the whole domain of masses and metallicities explored in the present work. Let us just mention here that, as will be described in Sect. 5, the critical velocity evolves with time, so the values given here are values at the beginning of the star's evolution and should not be used throughout the whole MS phase.

Figure 12 shows the relations between $v$ and $\omega$ on the ZAMS for different initial mass models at various metallicities. The corresponding values are also given in Table 2 . We see that, for a given value of $\omega$, higher surface velocities are obtained at lower metallicities and for higher initial masses.

\section{Evolution on the main sequence}

\subsection{Hertzsprung-Russell diagram and lifetimes}

Evolutionary tracks in the Hertzsprung-Russell diagram for the present models are shown in Fig. 13. The models have evolved until either reaching $\Omega_{\text {crit }}$ or just before the onset of central helium burning. For each mass, the effect of reducing the metal content shifts the tracks towards the blue part of the diagram and, for 3 and $9 M_{\odot}$, towards higher luminosities. In each metallicity subgroup, the effect of raising the rotational rate shifts the tracks towards lower luminosities and cooler temperatures on the ZAMS. When the star evolves, rotational mixing continuously refuels the convective core in fresh hydrogen and brings newly produced helium into the radiative zone. The enrichment of the radiative envelope in helium makes the star brighter and bluer by decreasing the opacity. If we look at what happens to the convective core (Fig. 14), we see that at first, a higher rotational rate leads to a smaller convective core, due to the lower central temperature. Then, we see that the decrease in the core is slower because of the refuelling in hydrogen. At the end of the MS, the core is much larger in the fast rotating models. In that respect rotation acts as a core overshoot.

In Table 3 the lifetimes of our stellar models are given, as well as the time at which the critical limit (if reached) is first encountered during the MS phase. For the models that were not pursued until the end of the MS phase, we give an estimate of the MS lifetime (indicated by an asterisk) obtained by inspections of the variation in the central hydrogen mass fraction with time. Note that we suppose that the star, once it reaches the critical limit, remains in the vicinity of this limit. This behaviour has been confirmed by previous computations in which models were pursued beyond the point where they first reached the critical limit (see Ekström et al. 2006). Most of the $60 M_{\odot}$ stellar models encounter the critical limit very early. In that case only 
Table 2. The first line indicates the equatorial velocity in $\mathrm{km} \mathrm{s}^{-1}$ on the ZAMS for each initial mass and metallicity. For the 3, 20, and $60 M_{\odot}$ stellar mass models, the second line gives the time-averaged value of the velocity over the adjustment period from solid to non-solid rotation at the beginning of the MS phase (see Sect. 4.2).

\begin{tabular}{|c|c|c|c|c|c|c|c|c|}
\hline \multirow[t]{2}{*}{$\overline{\overline{\mathrm{M}}}$} & \multirow[t]{2}{*}{$\overline{Z Z}$} & \multicolumn{7}{|c|}{$\bar{\Omega} \bar{\Omega} / \Omega_{\text {crit }}$} \\
\hline & & 0.1 & 0.3 & 0.5 & 0.7 & 0.8 & 0.9 & 0.99 \\
\hline 1 & 0 & 26 & 80 & 137 & 203 & 242 & 292 & 369 \\
\hline 1 & $10^{-5}$ & 26 & 80 & 137 & 202 & 242 & 291 & 369 \\
\hline 1 & 0.002 & 25 & 77 & 133 & 197 & 237 & 285 & 362 \\
\hline 1 & 0.020 & 26 & 78 & 134 & 199 & 237 & 286 & 362 \\
\hline \multirow[t]{2}{*}{3} & 0 & 40 & 121 & 210 & 312 & 367 & 438 & 548 \\
\hline & & 39 & 110 & 179 & 258 & 299 & 342 & 397 \\
\hline \multirow[t]{2}{*}{3} & $10^{-5}$ & 39 & 119 & 204 & 301 & 359 & 429 & 541 \\
\hline & & 38 & 107 & 174 & 249 & 293 & 340 & 391 \\
\hline \multirow[t]{2}{*}{3} & 0.002 & 34 & 103 & 178 & 262 & 312 & 375 & 473 \\
\hline & & 32 & 92 & 151 & 218 & 255 & 300 & 348 \\
\hline \multirow[t]{2}{*}{3} & 0.020 & 28 & 89 & 152 & 224 & 267 & 321 & 405 \\
\hline & & 29 & 83 & 137 & 199 & 231 & 269 & 312 \\
\hline \multirow[t]{2}{*}{9} & 0 & 62 & 188 & 322 & 473 & 564 & 678 & 860 \\
\hline & & 62 & 171 & 279 & 393 & 462 & 532 & 607 \\
\hline \multirow[t]{2}{*}{9} & $10^{-5}$ & 50 & 152 & 260 & 383 & 457 & 548 & 699 \\
\hline & & 48 & 132 & 220 & 322 & 378 & 444 & 511 \\
\hline \multirow[t]{2}{*}{9} & 0.002 & 44 & 128 & 220 & 325 & 387 & 465 & 589 \\
\hline & & 40 & 111 & 186 & 269 & 316 & 371 & 421 \\
\hline \multirow[t]{2}{*}{9} & 0.020 & 37 & 113 & 195 & 287 & 343 & 411 & 541 \\
\hline & & 36 & 102 & 169 & 247 & 291 & 340 & 384 \\
\hline \multirow[t]{2}{*}{20} & 0 & 85 & 256 & 434 & 644 & 766 & 918 & 1147 \\
\hline & & 77 & 214 & 349 & 512 & 584 & 672 & 769 \\
\hline \multirow[t]{2}{*}{20} & $10^{-5}$ & 58 & 177 & 303 & 447 & 532 & 639 & 819 \\
\hline & & 55 & 156 & 263 & 386 & 454 & 535 & 626 \\
\hline \multirow[t]{2}{*}{20} & 0.002 & 49 & 150 & 258 & 380 & 454 & 545 & 686 \\
\hline & & 45 & 129 & 221 & 323 & 379 & 444 & 515 \\
\hline \multirow[t]{2}{*}{20} & 0.020 & 45 & 136 & 233 & 344 & 409 & 490 & 639 \\
\hline & & 41 & 118 & 200 & 291 & 342 & 397 & 459 \\
\hline \multirow[t]{2}{*}{60} & 0 & 106 & 316 & 540 & 795 & 947 & 1129 & 1423 \\
\hline & & 97 & 276 & 476 & 688 & 827 & 952 & 1140 \\
\hline \multirow[t]{2}{*}{60} & $10^{-5}$ & 75 & 225 & 385 & 566 & 674 & 807 & 1017 \\
\hline & & 71 & 213 & 364 & 533 & 632 & 751 & 880 \\
\hline \multirow[t]{2}{*}{60} & 0.002 & 63 & 191 & 326 & 480 & 577 & 687 & 879 \\
\hline & & 57 & 175 & 300 & 440 & 517 & 609 & 708 \\
\hline \multirow[t]{2}{*}{60} & 0.020 & 56 & 171 & 292 & 428 & 509 & 603 & 732 \\
\hline & & 50 & 157 & 268 & 389 & 458 & 534 & 600 \\
\hline
\end{tabular}

the time of reaching the limit is given. We see that for $\omega=0.7$ the MS lifetime is increased by $15-23 \%$ (depending on the initial mass and metallicity) in comparison with the models with $\omega=0.1$.

\subsection{Evolution of the equatorial velocity}

The evolution of the rotation velocities at the stellar surface directly depends, of course, on the expansion/contraction movements of the star. But besides this, it also strongly depends on two factors: the internal mechanism of angular momentum transport (internal coupling) and the mass loss.

An extreme case of internal angular momentum transport is the one that imposes solid body rotation at each moment in the course of the evolution of the star. A strong coupling is then realised between the contracting core and the expanding envelope. The spinning up of the central regions is transmitted to the

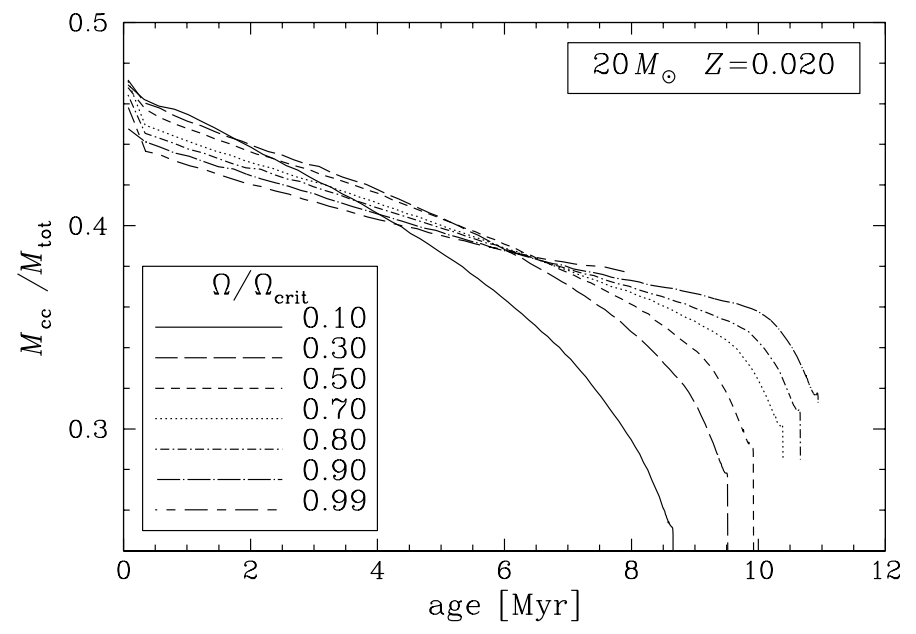

Fig. 14. Evolution in the size of the convective core during MS, for the various values of rotational rate, in the $20 M_{\odot}$ at standard metallicity.

stellar surface. Thus, during the MS phase, the surface velocity approaches the critical limit and may even reach it, depending on the initial conditions (see Sackmann \& Anand 1970; Langer 1997; Meynet et al. 2007). Another extreme case is the one of no transport of angular momentum. Each stellar layer keeps its own angular momentum. The variation in $\Omega$ is then simply governed by the local conservation of the angular momentum and, at the surface, $\Omega \sim R^{-2}$. When the radius increases, the surface angular velocity decreases more rapidly than the classical critical angular velocity (see Eq. (5)), thus the star evolves away from the critical limit. In the present models, without magnetic fields, the situation lies between these two extreme cases. A moderate coupling is mainly exerted by meridional circulation, which is more efficient than shear transport, as far as transport of angular momentum is concerned (see e.g. Meynet \& Maeder 2000).

The second significant effect governing the evolution of angular momentum is mass loss. The strong mass losses during the MS phase, expected for high-mass stars at high metallicities, decrease the angular momentum of the stars. The strength of this decrease in the angular momentum depends on how matter is ejected. In the extreme case where all the matter would be ejected along narrow jets centred on the rotational axis, nearly no angular momentum would be lost, while if all the matter is ejected near the equator plane, this maximizes the amount of angular momentum lost. Depending on the geometry of the wind, the surface velocity may thus decrease or increase. In the present work, we did not account for the effects of wind anisotropies (which would be strong only for a relatively narrow range of masses and velocities, see Meynet \& Maeder 2003), thus, in the present case, strong mass loss always leads to a decrease in the surface velocity.

Figure 15 shows the evolution of the surface velocity expressed as a fraction of the critical velocity during the MS phase for $20 M_{\odot}$ stellar models. Quite generally the curves can be decomposed into three parts, whose importance depends on the initial rotation and metallicity:

1. At the very beginning, there is a short adjustment period, which lasts for a few percent of the MS lifetime, during which meridional circulation transports angular momentum from the outer parts of the star to the inner ones. This slows down the surface of the star. Then, in the interior, shear turbulence becomes active and erodes the gradients built by the meridional circulation. Under the influence of these 
Table 3. Lifetimes (in Myr) during the MS phase. An asterisk means that the value was estimated (see text).

\begin{tabular}{|c|c|c|c|c|c|c|c|}
\hline \multirow[t]{2}{*}{$\overline{\bar{Z}}$} & \multicolumn{7}{|c|}{ 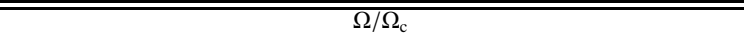 } \\
\hline & 0.1 & 0.3 & 0.5 & 0.7 & 0.8 & 0.9 & 0.99 \\
\hline \multicolumn{8}{|c|}{$3 M_{\odot}$} \\
\hline 0 & 215.6 & 227.8 & 237.8 & $\begin{array}{c}249 * \\
(223.7)\end{array}$ & $\begin{array}{c}255^{*} \\
(206.2)\end{array}$ & $\begin{array}{c}264 * \\
(189.0)\end{array}$ & $\begin{array}{c}275^{*} \\
(170.6)\end{array}$ \\
\hline $10^{-5}$ & 224.9 & 243.6 & & 261.5 & 265.6 & $\begin{array}{c}271 * \\
(270.6)\end{array}$ & $\begin{array}{c}271^{*} \\
(213.4)\end{array}$ \\
\hline 0.002 & 274.2 & 301.3 & 312.3 & 320.9 & 325.4 & $\begin{array}{c}331 * \\
(316.4)\end{array}$ & $\begin{array}{c}331 * \\
(237.9)\end{array}$ \\
\hline 0.020 & 357.0 & 399.6 & 424.4 & 439.2 & 444.4 & $\begin{array}{c}454 * \\
(446.7)\end{array}$ & $\begin{array}{c}484^{*} \\
(302.9)\end{array}$ \\
\hline \multicolumn{8}{|c|}{$9 M_{\odot}$} \\
\hline 0 & 20.31 & 22.11 & 23.24 & 24.31 & 24.74 & $\begin{array}{c}25^{*} \\
(24.77)\end{array}$ & $\begin{array}{c}25^{*} \\
(16.12)\end{array}$ \\
\hline $10^{-5}$ & 26.62 & 28.70 & 29.98 & 30.85 & 31.29 & $\begin{array}{c}31.8^{*} \\
(31.30)\end{array}$ & $\begin{array}{c}32.4^{*} \\
(25.03)\end{array}$ \\
\hline 0.002 & 27.77 & 30.11 & 31.32 & 32.26 & 32.87 & $\begin{array}{c}33.5^{*} \\
(31.84)\end{array}$ & $\begin{array}{c}34.0^{*} \\
(26.00)\end{array}$ \\
\hline 0.020 & 27.59 & 30.33 & 31.62 & 32.62 & $\begin{array}{c}33.0^{*} \\
(32.91)\end{array}$ & $\begin{array}{l}34.0^{*} \\
(28.89)\end{array}$ & $\begin{array}{c}34.0 * \\
(23.05)\end{array}$ \\
\hline \multicolumn{8}{|c|}{$20 M_{\odot}$} \\
\hline 0 & 8.003 & 8.554 & 8.868 & 9.199 & 9.324 & 9.434 & $\begin{array}{c}9.44 * \\
(8.616)\end{array}$ \\
\hline $10^{-5}$ & 9.099 & 9.853 & 10.213 & $\begin{array}{c}10.54 * \\
(10.533)\end{array}$ & $\begin{array}{l}10.68 * \\
(9.626)\end{array}$ & $\begin{array}{l}10.73 * \\
(7.843)\end{array}$ & $\begin{array}{l}10.73^{*} \\
(5.651)\end{array}$ \\
\hline 0.002 & 9.303 & 10.117 & 10.527 & $\begin{array}{c}10.85^{*} \\
(10.840)\end{array}$ & $\begin{array}{c}11.00^{*} \\
(10.033)\end{array}$ & $\begin{array}{l}11.06^{*} \\
(8.444)\end{array}$ & $\begin{array}{l}11.15^{*} \\
(6.712)\end{array}$ \\
\hline 0.020 & 8.663 & 9.521 & 9.928 & $\begin{array}{c}10.39^{*} \\
(10.389)\end{array}$ & $\begin{array}{c}10.66^{*} \\
(10.662)\end{array}$ & $\begin{array}{c}10.96^{*} \\
(10.937)\end{array}$ & $\begin{array}{l}10.96^{*} \\
(7.892)\end{array}$ \\
\hline \multicolumn{8}{|c|}{$60 M_{\odot}$} \\
\hline 0 & 3.633 & 3.802 & 3.924 & (3.386) & (2.538) & (2.259) & (1.173) \\
\hline $10^{-5}$ & 3.874 & 4.112 & (3.696) & $(2.746)$ & $(2.200)$ & $(1.525)$ & $(0.783)$ \\
\hline 0.002 & 3.994 & 4.251 & $(4.432)$ & (2.818) & (2.336) & (1.717) & (1.087) \\
\hline 0.020 & 3.714 & 3.978 & $(3.960)$ & $(2.426)$ & (2.031) & (1.595) & (1.179) \\
\hline
\end{tabular}

two counteracting effects, the $\Omega$-profile converges toward an equilibrium configuration (Zahn 1992; Denissenkov et al. 1999; Meynet \& Maeder 2000). From Fig. 15, one sees that for lower initial values of $v / v_{\text {crit }}$ the decrease is much weaker than for higher values. It may even completely disappear. It illustrates the dependence of the meridional velocity on $\Omega$. This feature also shows that, in the present theoretical context, we cannot have stars that would remain near the critical limit starting from the ZAMS. Indeed, our most extreme case, the model with $\Omega / \Omega_{\text {crit }}=0.99$ evolves away from the critical limit just after the ZAMS.

Of course we can wonder to what extent this behaviour is dependent on the chosen initial conditions. For instance would this behaviour be the same, if one had computed realistic pre-MS evolution? Probably in that case the model would not have solid body rotation on the ZAMS and thus the above feature is in part artificial. It is difficult to say more, however, due to the complexity of the processes affecting the angular momentum of stars during their formation process. The point to keep in mind is that we cannot have non-evolved critically rotating stars in the present models. Let us stress that these stars should be very rare and their absence should not have any impact on the frequency of stars predicted to rotate at or near the critical velocity. The above effect also implies
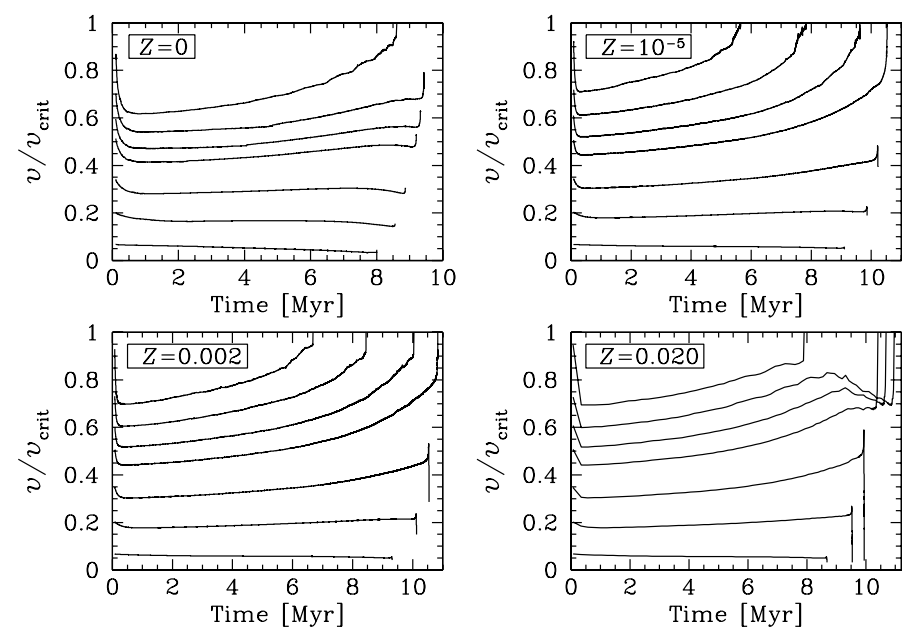

Fig. 15. Evolution of the ratio $v / v_{\text {crit }}$ during the core hydrogen-burning phase for the $20 M_{\odot}$ models at various metallicities. The computations were stopped as soon as the star reaches the critical limit or just before the beginning of the core He-burning phase.

that the velocities on the ZAMS given in Table 2 are probably quantities not directly comparable to observed ones. Indeed, in the most optimistic case, where ZAMS models indeed rotate as solid body, the chance of seeing stars at the very beginning of their relaxation period is very low. More realistic values of the initial velocities would correspond to time-averaged values computed over the period during which about ten percent of the initial content in hydrogen has been burnt in the core. These values are also given in Table 2. It is important to note that models treating advection as a diffusion would miss this first relaxation period. Indeed during this phase, meridional currents build the gradient of $\Omega$, a process that diffusion cannot do, since it always erodes any gradient.

2. As explained for instance in Maeder \& Meynet (2000b), after the first adjustment period, a large outer cell of meridional circulation sets in, transporting angular momentum from the inner parts of the star to the outer ones ${ }^{4}$. If this transport is rapid enough and the stellar winds not too intense, then $v / v_{\text {crit }}$ increases. (An essential distinction has to be made here: it is the ratio $v / v_{\text {crit }}$ that increases, not necessarily the equatorial velocity itself, see Fig. 18 and Sect. 5 for discussion on this point.) This is the case for all the models with $\omega \geq 0.3$ at $Z \geq 0.00001$. For metal-free stars, the increase in $v / v_{\text {crit }}$ only occurs for the models with $\omega \geq 0.7$. This is a consequence of the Gratton-Öpik effect (see the term in brackets in Eq. (14)) making the meridional circulation velocity lower at lower $Z$.

3. At the end of the MS phase, the increase of $v / v_{\text {crit }}$ accelerates when the star contracts after the blue hook in the HR diagram. The rapid change of the velocity at that stage is due to the rapid change in the radius, not to any transport mechanisms.

Looking at the right bottom panel of Fig. 15 (models at $Z=$ 0.020 ), another interesting effect can be discussed. We see for

\footnotetext{
${ }^{4}$ In our model, we have two cells of meridional circulation during the MS phase, an inner one where matter moves clockwise and an outer one where matter moves counter-clockwise. The velocities in the outer cell are much higher than the velocities of the inner cell. As a consequence, the outer cell clearly dominates the transport of the angular momentum in the star, transporting it from inner regions towards outer ones.
} 

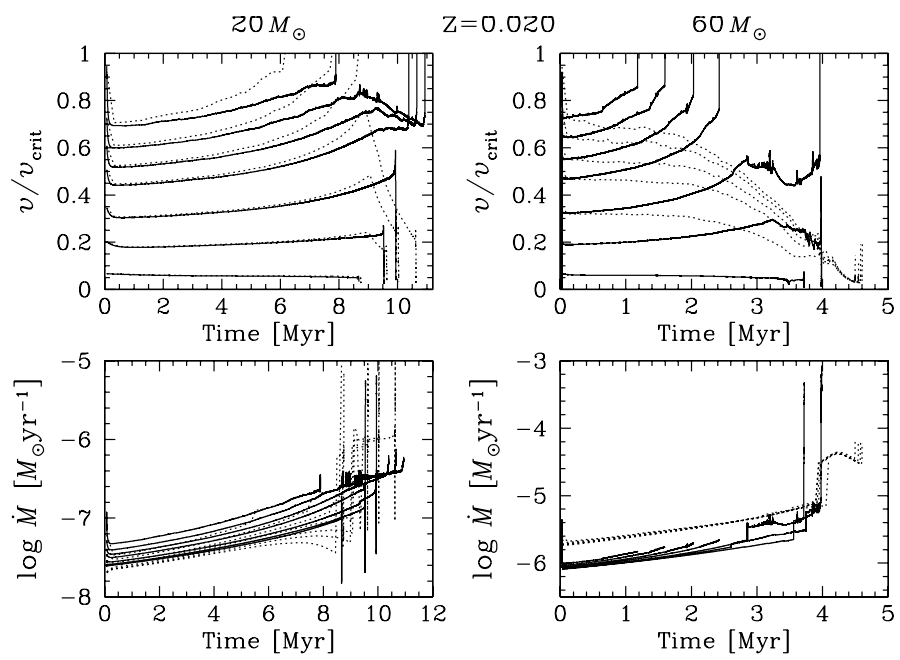

Fig. 16. Comparison of the evolution of $v / v_{\text {crit }}$ (top) and $\log \dot{M}$ (bottom) for the $20 M_{\odot}$ models (left) and the $60 M_{\odot}$ models (right) at standard metallicity, when using Kudritzki \& Puls (2000) (continuous lines) or Vink et al. (2000) (dotted lines).

instance that the $20 M_{\odot}$ stellar models with an initial value of $v / v_{\text {crit }}$ between 0.5 and 0.7 ( $\omega$ between 0.7 and 0.9 ) show a bump around the age of $9 \mathrm{Myr}$. This occurs because, at that time for these models, mass loss increases enough to efficiently remove the angular momentum brought to the surface by the meridional currents. The surface velocity thus evolves away from the critical limit, reaching it again only at the very end of the MS phase. This feature is not seen at lower metallicity because mass loss rates are lower. This trend was already shown by Maeder \& Meynet (2001). For a given degree of coupling, the mass loss rates play a very critical role in the evolution of the surface rotation.

In order to check the validity of our results, we computed another set of 20 and $60 M_{\odot}$ models at $Z=0.020$ with the mass loss prescription of Vink et al. (2000, hereafter VdKL00). In the case of the $20 M_{\odot}$ models, the mass loss rates obtained in the new set are slightly lower than the ones obtained with Kudritzki $\&$ Puls (2000, KP00). The $\omega \geq 0.8$ models thus meet the breakup limit earlier in their evolution. The $\omega=0.7$ model also shows a bump in the velocity, as described above, but then it stays far from the break-up limit, even at the end of the MS, unlike the case with the KPO0 rates. This leads to the minimum $\omega$ required to reach the break-up limit during evolution being slightly higher with VdKL00 rates, while the fast rotators spend a little more time at the break-up limit (Fig. 16, left panels). But the qualitative result is generally similar to the one obtained with KP00 rates. In the $60 M_{\odot}$ case, the opposite happens: VdKL00's rates are much stronger (by a factor 2 during the MS), and thus no model reaches the break-up limit anymore, even the one starting its evolution with $\omega=0.99$ (Fig. 16, right panels). The most massive stars' evolution is dramatically influenced by the choice of the mass loss rates, and it can be concluded that, in that case, the comparison between theoretical models and observations instead constitutes a check on the mass loss rates more than anything else.

Coming back to our initial set of models, we present in Table 4 the time-averaged velocities during the MS phase or during the period before the reaching of the critical limit. This table is useful for connecting the values of $\omega$ on the ZAMS with observable rotational properties during the MS phase (see Sect. 5).
Table 4. Time-averaged equatorial velocities in $\mathrm{km} \mathrm{s}^{-1}$ corresponding to various ZAMS values of $\omega=\Omega / \Omega_{\text {crit }}$. The average is taken either on the whole MS phase or on the part of the evolution before the critical limit is reached. For these latter models, the mass fraction of hydrogen at the centre when the surface velocity reaches the critical limit is indicated in parenthesis.

\begin{tabular}{|c|c|c|c|c|c|c|c|c|}
\hline \multirow[t]{2}{*}{$\overline{\bar{M}}$} & \multirow[t]{2}{*}{$Z$} & & & $\omega$ & $=$ & $\Omega / \Omega_{\text {crit }}$ & & \\
\hline & & 0.1 & 0.3 & 0.5 & 0.7 & 0.8 & 0.9 & 0.99 \\
\hline 3 & 0 & 38 & 108 & 198 & $\begin{array}{c}294 \\
(5 e-2)\end{array}$ & $\begin{array}{c}333 \\
(0.1)\end{array}$ & $\begin{array}{c}373 \\
(0.17)\end{array}$ & $\begin{array}{c}423 \\
(0.26)\end{array}$ \\
\hline 3 & $10^{-5}$ & 33 & 95 & 167 & 247 & 297 & $\begin{array}{c}353 \\
(7 e-3)\end{array}$ & $\begin{array}{c}416 \\
(0.30)\end{array}$ \\
\hline 3 & 0.002 & 27 & 80 & 139 & 207 & 246 & $\begin{array}{c}298 \\
(0.1)\end{array}$ & $\begin{array}{c}352 \\
(0.38)\end{array}$ \\
\hline 3 & 0.020 & 23 & 69 & 120 & 181 & 215 & $\begin{array}{c}258 \\
(3 e-2)\end{array}$ & $\begin{array}{c}311 \\
(0.38)\end{array}$ \\
\hline
\end{tabular}

$\begin{array}{ccccccccc}9 & 0 & 56 & 151 & 265 & 394 & 477 & 569 & 666 \\ 9 & 10^{-5} & 39 & 117 & 204 & 305 & 363 & \begin{array}{c}435 \\ 435\end{array} & \begin{array}{c}515 \\ (3 \mathrm{e}-2)\end{array} \\ (0.29) \\ 9 & 0.002 & 33 & 99 & 173 & 256 & \begin{array}{c}305 \\ 369\end{array} & 425 \\ (3 \mathrm{e}-4) & (9 \mathrm{e}-2) & (0.31) \\ 9 & 0.020 & 30 & 90 & 157 & 236 & \begin{array}{c}284 \\ (2 \mathrm{e}-2)\end{array} & \begin{array}{c}341 \\ (0.21)\end{array} & \begin{array}{c}387 \\ (0.36)\end{array}\end{array}$

$\begin{array}{ccccccccc}20 & 0 & 54 & 169 & 295 & 446 & 507 & 588 & 721 \\ & & & & & & & & (0.13) \\ 20 & 10^{-5} & 45 & 144 & 254 & 389 & 469 & 557 & 651 \\ & & & & & (3 \mathrm{e}-3) & (0.15) & (0.33) & (0.48) \\ 20 & 0.002 & 37 & 120 & 213 & \begin{array}{c}323 \\ 387\end{array} & 456 & 528 \\ & & & & & (2 \mathrm{e}-3) & (0.14) & (0.30) & (0.43) \\ 20 & 0.020 & 32 & 107 & 189 & \begin{array}{c}279 \\ (2 \mathrm{e}-4)\end{array} & \begin{array}{c}325 \\ (1 \mathrm{e}-4)\end{array} & \begin{array}{c}369 \\ (6 \mathrm{e}-4)\end{array} & (0.30) \\ & & & & & & & & \end{array}$

\begin{tabular}{ccccccccc}
60 & 0 & 63 & 248 & 456 & 645 & 830 & 930 & 1167 \\
& & & & & $(0.20)$ & $(0.38)$ & $(0.45)$ & $(0.61)$ \\
60 & $10^{-5}$ & 63 & 221 & 408 & 587 & 687 & 797 & 890 \\
& & & & $(0.16)$ & $(0.37)$ & $(0.47)$ & $(0.57)$ & $(0.67)$ \\
60 & 0.002 & 48 & 179 & 323 & 474 & 552 & 641 & 727 \\
& & & & $(5 \mathrm{e}-5)$ & $(0.40)$ & $(0.45)$ & $(0.54)$ & $(0.63)$ \\
60 & 0.020 & 37 & 140 & $\begin{array}{c}258 \\
(8 \mathrm{e}-2)\end{array}$ & $\begin{array}{c}400 \\
(0.38)\end{array}$ & $\begin{array}{c}472 \\
(0.44)\end{array}$ & $\begin{array}{c}547 \\
(0.51)\end{array}$ & $\begin{array}{c}609 \\
\end{array}$ \\
\hline
\end{tabular}

\subsection{Surface enrichment}

In Fig. 17 the tracks are plotted in the plane $(\mathrm{N} / \mathrm{C}) /(\mathrm{N} / \mathrm{C})_{\text {ini }}$ versus $P$, where $P$ is the rotational period in hours. During the evolution the surface is progressively enriched in CNO-burning products, i.e. enriched in nitrogen and depleted in carbon. At the same time, the rotational period increases. The hatched regions correspond to zones where stars would rotate at or faster than the critical limit. We note the following features:

- As already mentioned in previous works (see e.g. Maeder \& Meynet 2001), for a given value of the initial mass, of $\omega$, and for a given evolutionary stage, the surface enrichments are stronger in metal-poor stars. This is related mainly to the fact that the gradients of $\Omega$ are steeper in metal poor stars due the slower meridional currents in more compact stars. We note that surface enrichments for $3 M_{\odot}$ stellar models at $Z=0.02$ remain in the range of a few percent, even for high rotation rates. This means that for these low mass stars, observations 
will probably not be able to see such small enrichments. The same initial mass models at $Z=0.002$ present enhancements of up to $45 \%$.

- The surface enrichments are also higher in more massive stars, a feature that is due to the higher efficiency of the shear mixing when the initial mass increases.

- Stars with higher initial velocities reach higher N/C ratios. Let us recall here that the computations were stopped when the critical limit was reached, explaining why, for instance, the maximum surface enrichment indicated for the $9 M_{\odot}$ stellar model at $Z=0.02$ is lower for $\Omega / \Omega_{\text {crit }}=0.99$ than for $\Omega / \Omega_{\text {crit }}=0.8$ and 0.9 . If the model with $\Omega / \Omega_{\text {crit }}=0.99$ were continued, it would reach higher values than those shown in Fig. 17.

- In general, stars near the critical limit show nitrogen enhancements. There are, however, cases where no enhancements or very small ones would be expected. These cases are the stars starting their evolution with very high rotation rates and still being nearly non-evolved. For instance, in the case of the $9 M_{\odot}$ stellar model at $Z=0.02$ with $\Omega / \Omega_{\text {ini }}=0.99$, $v / v_{\text {crit }}$ becomes superior to 0.7 , while still no significant surface enrichment in nitrogen has occurred. Another case where no observable surface enrichment is expected is for stars at $Z=0.02$ and with initial masses around $3 M_{\odot}$ (see lower left panel).

- No stars with masses between 3 and $20 M_{\odot}$ and with rotation periods longer than about 5 days are expected to be at the critical limit.

\section{Initial conditions for stars reaching nearly critical velocities}

Let us first recall that a key ingredient of the ratio $v / v_{\text {crit }}$ is the behaviour of $v_{\text {crit }}$ itself. Figure 18 shows how it evolves in time in the case of the $20 M_{\odot}$ model at standard metallicity with $\omega=0.7$ initially $\left(v / v_{\text {crit }}=0.5\right)$. During evolution, $v_{\text {crit }}$ gets lower, and in the present case the final value amounts to only half the initial one. It can be explained by the modifications of the stellar parameters: some mass is lost and the radius is slowly inflating, so the surface gravity decreases and can be more easily counterbalanced by the centrifugal force. In the example shown in Fig. 18, we see that, even though the equatorial velocity is slowly decreasing during the MS, the ratio $v / v_{\text {crit }}$ increases steadily. It is essential to take this evolutionary effect into account when one wants to determine the $v / v_{\text {crit }}$ ratio at any given time. Keeping the initial value would lead to a completely wrong result. Let us mention that, in that perspective, our results are not contradictory with the observations of Huang \& Gies (2006b) or Abt (2003), who find a clear spin down with evolution for the stars in their sample. Except the most extreme ones with $\omega=0.99-0.90$, all our standard metallicity models in the mass range $3-9 M_{\odot}$ show a steady or decreasing equatorial velocity, though their $v / v_{\text {crit }}$ ratios increase.

From our computations, we can determine, for each model specified by its initial mass and metallicity, the values of $\Omega / \Omega_{\text {crit }}$ on the ZAMS required to reach given limits of $v / v_{\text {crit }}$ during the MS phase. These values are plotted in Fig. 19. Using Table 2, it is possible to translate these limits on $\Omega / \Omega_{\text {crit }}$ into limits on the equatorial velocities on the ZAMS or at the beginning of the MS phase. Using Table 4, a similar correspondence can be obtained with values of the averaged velocity during the MS phase or during the phase before reaching the critical velocity.

At the standard metallicity, we note that in general the values of $\Omega / \Omega_{\text {crit }}$ needed on the ZAMS to reach given values of $v / v_{\text {crit }}$ decrease when the mass increases. This comes from the fact that meridional currents are more rapid in the less dense envelope of more massive stars. Let us recall that, in the outer layer, the dominant term in the expression for the radial component of the meridional circulation $U(r)$ is mainly (Gratton-Öpik term)

$U(r) \sim\left[1-\frac{\Omega^{2}}{2 \pi G \bar{\rho}}\right]\left(\frac{\Omega^{2} r^{3}}{G M}\right)$

where $\bar{\rho}$ means the average over the considered equipotential. Thus the outer cell of the meridional circulation will be more active in less dense envelopes. In the upper left panel of Fig. 19, the bump around the $20 M_{\odot}$ stellar model comes from this model suffering significant mass loss at the end of its MS evolution. This discourages reaching nearly critical values (except at the very end of the MS evolution, see Fig. 15). In the case of the $60 M_{\odot}$ model, when the mass loss rates of KP00 are used, the nearly critical values occur well before the star undergoes similar enhancements of the mass loss rate, and thus, the minimum values of $\Omega / \Omega_{\text {crit }}$ are lower than for the $20 M_{\odot}$. The situation is quite different if the mass loss rates from VdKL00 are used (see Fig. 16).

For metallicities $Z=0.002$ and 0.00001 , we find that the minimum values of $\Omega / \Omega_{\text {crit }}$ required to reach near critical velocities also decrease when the initial mass increases. More massive stars thus reach the critical limit more easily. We also note that for the $60 M_{\odot}$ at $Z=0.002$, the minimum value of $\Omega / \Omega_{\text {crit }}$ to reach the critical velocity is around 0.7 , while at $Z=0.00001$ it is lowered to values equal to 0.5 . This is mainly due to the weak radiative winds of the metal-poor models. Since radiative winds are triggered by metal lines, a low metal content implies low mass loss rates. We have already seen in Sect. 4.2 that there is a competition between the efficiency of the mass loss (removing some angular momentum and thus decelerating the surface) and the efficiency of the meridional circulation (bringing the angular momentum from the core to the surface and thus accelerating the surface). From our results, we can say that, even though the efficiency of the meridional circulation is lower at low $Z$, the effect of lowering the mass loss rates plays the major role and the total budget favours a significant acceleration of the surface, at least down to $Z=0.00001$. Let us note that at some points, the curves are superposed: for instance for the $9 M_{\odot}$ at $Z=0.002$, the curve for reaching the critical limit and those for reaching values of $v / v_{\text {crit }} \geq 0.8$ and 0.9 go through the same point. This simply reflects the fact that, when such a star reaches the values $v / v_{\text {crit }} \geq 0.8$, it then also reaches the critical limit.

For metal-free stars, one notes that, for $M>9 M_{\odot}$, the minimum values of $\Omega / \Omega_{\text {crit }}$ to approach critical velocities during the MS evolution are in general higher than at higher metallicities. This reflects the greater compactness of these stars. The metalfree $3 M_{\odot}$ models, in contrast reach the critical limit much more easily than the models at higher metallicities. We can see that their tracks in the HR diagram (see Fig. 13) evolve quite blueward. This evolution is due to the fact that metal-free stars in this mass domain must contract over a large part of their MS evolution to compensate for the lack of CNO elements. In such a situation, the evolution of $\Omega$ is mainly governed by the local conservation of the angular momentum (nearly no coupling), which favours the approach of the critical limit when the star contracts. Typically for a $3 M_{\odot}$ stellar model, the minimum value of $\Omega / \Omega_{\text {crit }}$ is around 0.6 at $Z=0$ for reaching the critical limit during the MS phase, while it is higher than 0.8 at standard metallicity. 

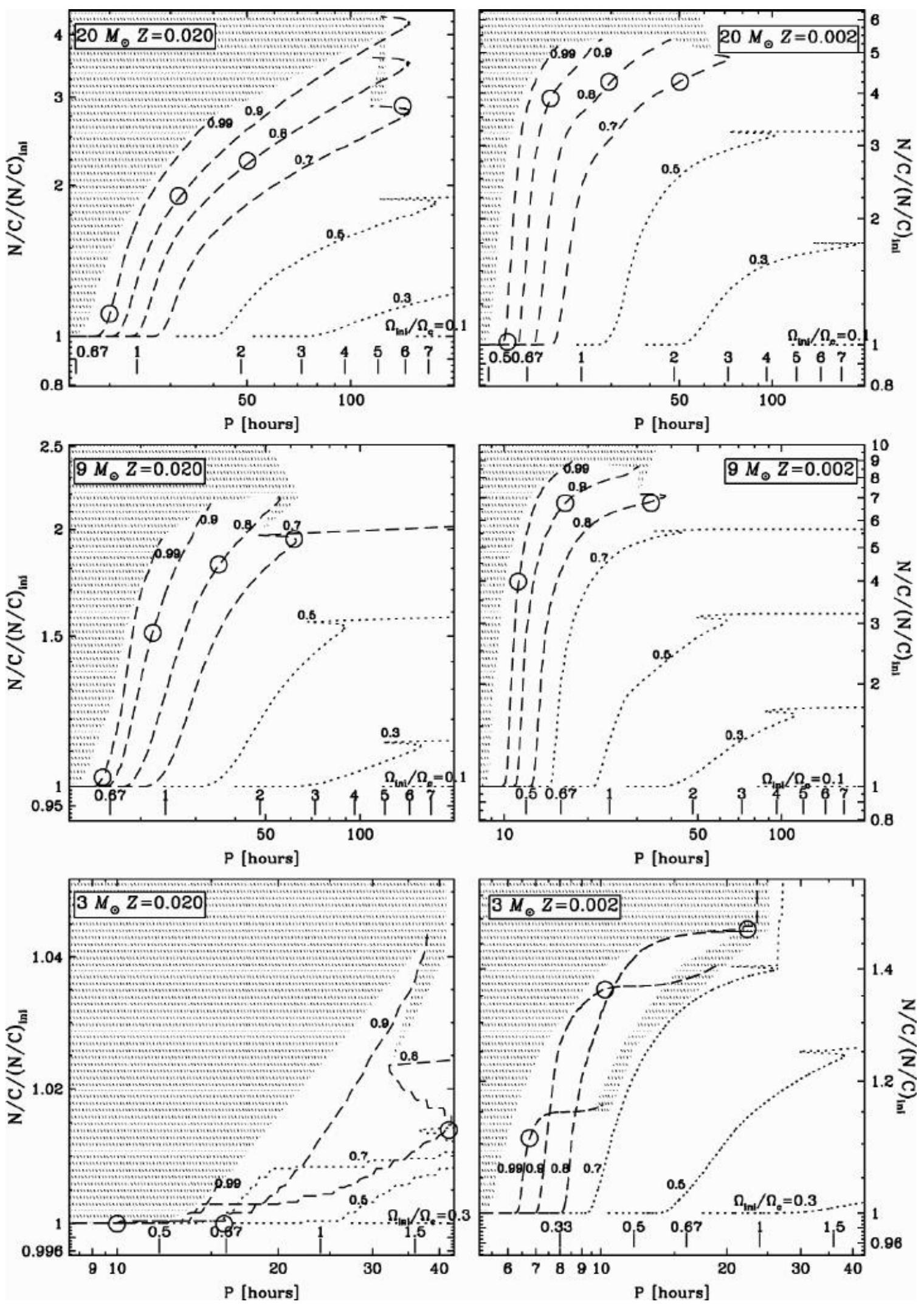

Fig. 17. Evolutionary tracks in the plane surface N/C ratio, normalized to its initial value, versus the rotational period in hours for different initial mass stars, various initial velocities, and the metallicities $Z=0.02$ and 0.002 . Positions of some periods in days are indicated at the bottom of the figure. The dotted tracks never reach the critical limit during the MS phase. The short dashed tracks reach the critical limit during the MS phase. The dividing line between the shaded and non-shaded areas corresponds to the entrance into the stage when the star is at the critical limit during the MS evolution. Big circles along some tracks indicate the stage when $v / v_{\text {crit }}$ becomes superior to 0.7. If Be stars are rotating at velocities superior to $70 \%$ of the critical velocity, the present models would predict that they would lie in the region between the big circles and the dividing line. Beware the different vertical scales used when comparing similar masses at different metallicities. 


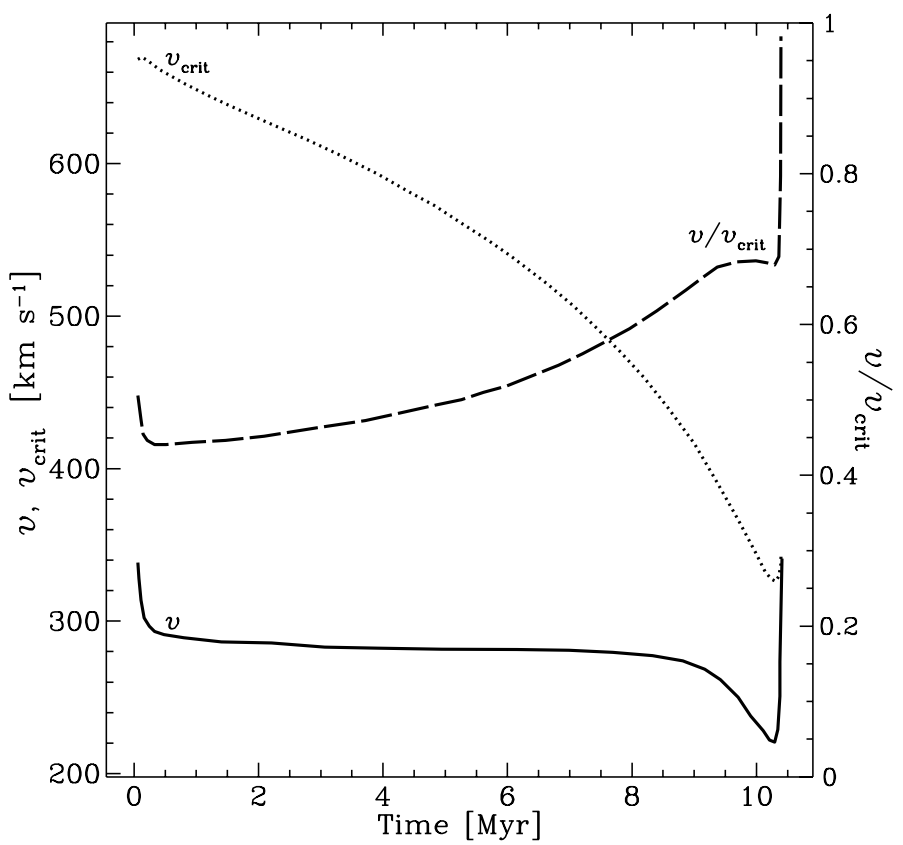

Fig. 18. Evolution of the equatorial velocity $v$ (continuous line), the critical velocity $v_{\text {crit }}$ (dotted line), and their ratio (dashed line) for the $20 M_{\odot}$ at standard metallicity with $\omega=0.70$ initially $\left(v / v_{\text {crit }}=0.50\right)$.

\section{Rotation of stars in clusters of various ages and metallicities}

Let us call $n(t, w)$ the number of MS stars in a cluster of age $t$ having surface rotational velocities, such that $v / v_{\text {crit }} \geq w$. We only consider stars brighter than about two magnitudes below the turn off and call $N(t)$ the total number of MS stars in the same cluster, in the same interval of magnitudes. The ratio $n(t, w) / N(t)$ can be expressed as

$$
\frac{n(t, w)}{N(t)}=\frac{\int_{M_{\min }}^{M_{\max }} \bar{n}(M) p(M, t, w) \mathrm{d} M}{\int_{M_{\min }}^{M_{\max }} \bar{n}(M) f(M, t) \mathrm{d} M}
$$

where $M_{\min }$ is the mass of stars that are at two magnitudes below the turn off, $M_{\max }$ the mass of stars at the turn off, $\bar{n}(M)$ the number of stars born with an initial mass $M$, and $p(M, t, w)$ the fraction of these stars that, at the age $t$, are on the MS and have surface velocities such that $v / v_{\text {crit }} \geq w, f(M, t)$ is the fraction of stars of initial mass $M$ that are on the MS at the time $t$. In the following, we explain how these different values are obtained from our rotating stellar models.

- $M_{\max }$ : In the case of non-rotating stellar models, at a given metallicity we have a one-to-one relation between the initial mass and the MS lifetime. It suffices then to take the initial mass value of the star that has a MS lifetime equal to the considered age of the cluster to obtain the mass of stars at the turn off, i.e. $M_{\max }$. When rotation is accounted for, the situation is more complicated, since the MS lifetimes increase with the initial velocities. Thus the maximum mass of a star that can still be on the MS at a given age corresponds to the mass of the rotating star with the maximum initial rotation velocity (here $\Omega / \Omega_{\text {crit }}=0.99$ on the ZAMS) and with MS lifetime that corresponds to the age considered. Of course in a given cluster there is little chance of having at turn off such a star due to the very small number of stars with such a high velocity. This effect will be taken into account through the initial velocity distribution function in $f(M, t)$ (see below).
- $M_{\min }$ : To estimate the lower bound $M_{\min }$, we have taken about $70 \%$ of the value of $M_{\max }$. We indeed checked, using theoretical isochrones (Meynet et al. 1993), that for ages between 8 and $316 \mathrm{Myr}$, the typical mass of stars two visual magnitudes below the turn off is in that range.

$-\bar{n}(M)$ : The number of stars born in the mass interval between $M_{\min }$ and $M_{\max }$ is proportional to $\int_{M_{\min }}^{M_{\max }} M^{-2.35} \mathrm{~d} M$, when a Salpeter's slope of the initial mass function is considered.

- $f(M, t)$ : as explained above, stars with a mass $M_{\max }$ will still be in their MS phase, provided they started their evolution with $\Omega / \Omega_{\text {crit }} \geq 0.99$. Stars of this mass but with slower initial rotation will have already evolved away from the MS. Thus the fraction of stars of mass $M_{\max }$ that are still on the MS at time $t$ is equal to

$$
f\left(M_{\max }, t\right)=\int_{\omega_{\min }(M, t)=0.99}^{\omega=1.00} d_{\mathrm{p}}(\omega) \mathrm{d} \omega
$$

where $\omega_{\min }(M, t)$ is the minimum value of $\omega$ for stars of initial mass $M$ to still be on the MS at time $t$, and $d_{\mathrm{p}}$ the probability density for a star to start its evolution with a given value $\omega$ (See Fig. 20). For each mass and age, one can determine $\omega_{\min }(M, t)$, and similar integrals as the one shown above can be performed.

- $p(M, t, w)$ : From figures similar to Fig. 15, we can extract, for each initial mass, metallicity, and rotation, the time at which $v / v_{\text {crit }}$ becomes superior to $w$ (note that once $v / v_{\text {crit }} \geq w$, it remains greater for the rest of the MS phase ${ }^{5}$, the only exception being the $20 M_{\odot}$ at $Z=0.020$ ). From such data, one can deduce that for a star of $9 M_{\odot}$ with $Z=0.020$ and an age equal to $25 \mathrm{Myr}$, the minimum value of $\Omega / \Omega_{\text {crit }}$ required on the ZAMS to reach values of $v / v_{\text {crit }}$ superior to 0.7 during the MS phase is 0.83 . Using an initial distribution of $\Omega / \Omega_{\text {crit }}$ (see Fig. 20), one can deduce the value of $p(M, t, w)$.

We now need to specify the function we use for the initial distribution of $\Omega / \Omega_{\text {crit }}$. Huang \& Gies (2006a) provide a distribution of equatorial velocities corrected for the $\sin i$ effect from their observations of 496 OB-type stars. This distribution is shown in Fig. 20 (left). Of course this distribution does not correspond to a distribution on the ZAMS, since stars are picked up at different phases of their evolution during the MS phase. We can reasonably consider, however, that these equatorial velocities are representative of the values for the average velocities of stars in the MS phase. We can then associate a value of $\omega$ on the ZAMS to each of these values using the relation obtained from our $9 M_{\odot}$ at standard metallicity between the averaged equatorial velocity during the MS phase and $\omega$ on the ZAMS. Proper normalization has been applied to transform the probability densities for velocities into probability densities for $\omega$, i.e. the normalization has been chosen so that $\int_{0}^{420} D_{\mathrm{p}}(v) \mathrm{d} v=\int_{0}^{1} d_{\mathrm{p}}(\omega) \mathrm{d} \omega=1$, where $D_{\mathrm{p}}$ and $d_{\mathrm{p}}$ are the probability densities for $v$ (see Fig. 20, left panel) and $\omega$, respectively. The cumulative distribution of $\omega$ on the ZAMS that we obtained in this way is shown in the right panel of Fig. 20.

In Fig. 21 the variation in the number fraction of stars at the critical limit (continuous thick lines) in clusters of different ages is shown. We also plotted the fraction of stars having $v / v_{\text {crit }}$ values superior to given limits (see caption). Due to the coarse grid of mass we used here, we shall not attach too much importance

\footnotetext{
5 Note that we used the extrapolated lifetimes (marked with an asterisk in Table 3) for the models whose computation was stopped before completing the MS phase.
} 

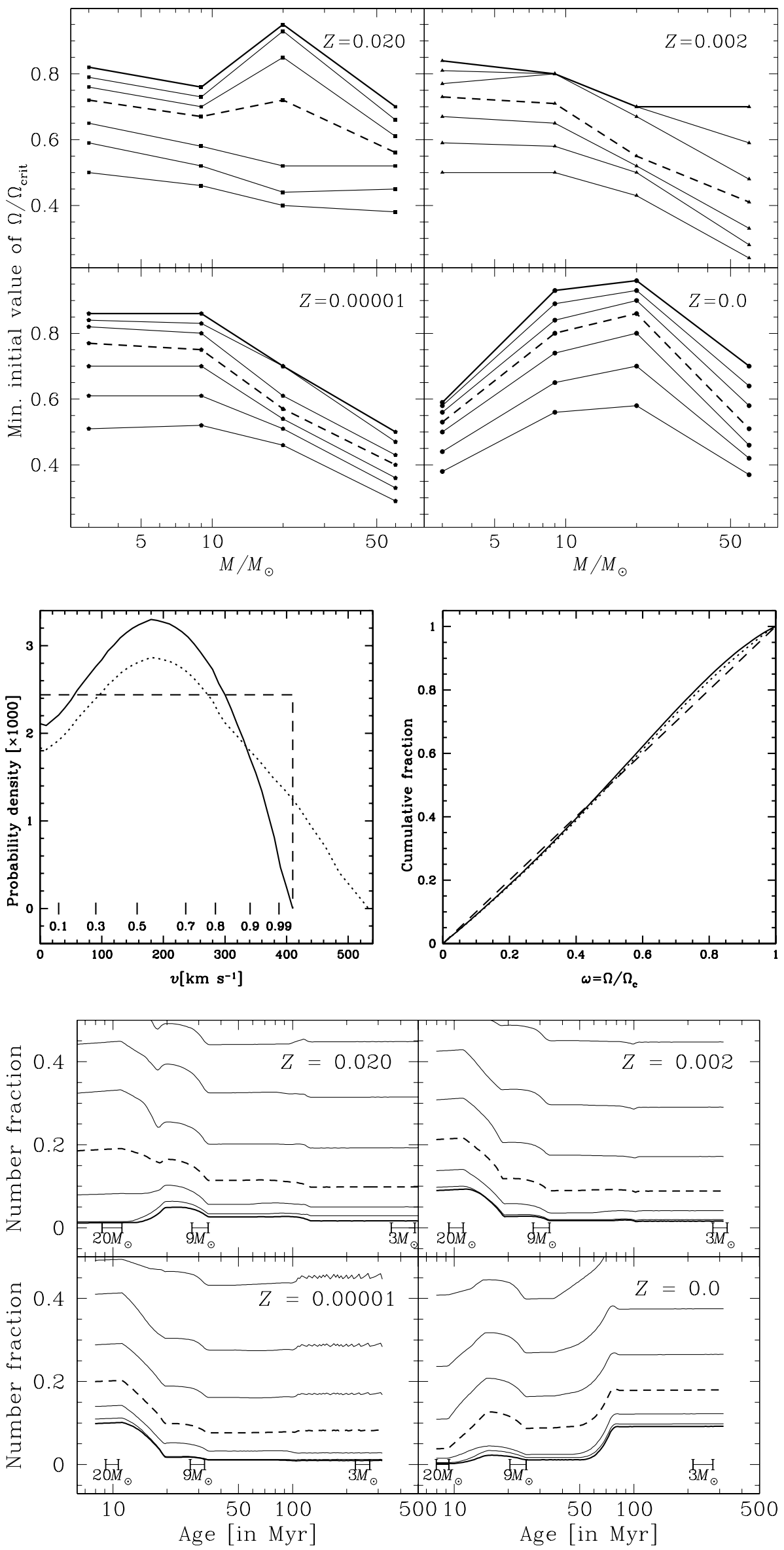

Fig. 19. Minimum values of $\omega=\Omega / \Omega_{\text {crit }}$ on the ZAMS required for reaching values of $v / v_{\text {crit }}$ superior to 0.4 (bottom line), 0.5, 0.6, 0.7 (dashed), 0.8, 0.9 and 0.99 (top heavy line), respectively, during the MS phase. The initial metallicity considered is indicated in each panel.

Fig. 20. Left panel: distribution of the equatorial velocities as given by Huang \& Gies (2006a) (continuous line). The dotted line shows the case when it is assumed that, above $300 \mathrm{~km} \mathrm{~s}^{-1}$, the velocities are underestimated (see text). The dashed line shows a flat distribution. At the bottom of the figure, the correspondence between ZAMS values of $\Omega / \Omega_{\text {crit }}$ and average velocities during the MS phase as deduced from our $9 M_{\odot}$ at standard metallicity (Table 4) is indicated. Right panel: cumulative distribution function of $\omega=\Omega / \Omega_{\text {crit }}$ deduced from the data by Huang \& Gies (2006a) with the same assertions as in the left panel.
Fig. 21. Variation in the fraction of stars with $v / v_{\text {crit }}$ superior or equal to $0.4,0.5,0.6$ (three thin continuous lines from the top), 0.7 (thick dashed line), 0.8, 0.9 (two thin continuous lines), and at the critical limit (bottom thick solid line) in clusters of various ages and metallicities. At the bottom of each panel, the range of MS lifetimes are indicated for the 3,9 and $20 M_{\odot}$ stellar models. 
to the details of these curves but will comment on their general behaviour:

- At standard metallicity, we note a bump around ages 20-25 Myr for the curves corresponding to the critical limit and for $v / v_{\text {crit }} \geq 0.9$ and 0.8 . This kind of behaviour can be explained in the following way: at younger ages than the bump, mass loss discourages reaching the critical limit, at older ages than the bump, meridional currents are not efficient enough for transporting the angular momentum outwards (denser envelopes in stars of lower initial masses).

- For metallicities $Z=0.002$ and 0.00001 , we note that the bump shifts to younger ages and reaches higher values. This is because of the lower mass loss rates experienced by stars at low metallicity. This allows more massive stars, which have less dense envelopes and thus stronger meridional currents, to undergo a more rapid acceleration of their surface and to reach the critical limit more easily.

- We note that the curves are very similar at the metallicities $Z=0.002$ and 0.00001 . Thus, from the point of view of the surface velocities, we would not expect any significant changes over this metallicity range.

- For metal-free stars, the curves are very different than at $Z=$ 0.00001 and 0.020: at ages younger than about 20-32 Myr, stars have more difficulty reaching the critical limit. This comes from the absence of metals making these stars very compact, with dense outer layers and very slow meridional circulation currents (note that if the mass range were extended towards higher masses, this bump might appear at very young ages). At ages older than about $80 \mathrm{Myr}$, on the other hand, stars at the critical limit are much more numerous than at higher metallicities. This comes from the fact that stars with initial masses equal or below $3-5 M_{\odot}$ contract for a large part of their MS phases (see the metal-free $3 M_{\odot}$ stellar models) and thus can reach the break-up limit quite early.

- At $Z=0.020$, the fraction of stars at the critical limit is at most $5 \%$ and can reach values of slightly more than $10 \%$ at lower metallicities. The number fraction of stars with $v / v_{\text {crit }} \geq 0.9$ tightly follows the curve of stars at the critical limit, indicating, that when this value is reached, the surface velocity rapidly evolves towards the critical limit. The fraction of stars with $v / v_{\text {crit }} \geq 0.7$ reaches values up to $20-25 \%$ and does so at an earlier age: around $10 \mathrm{Myr}$ and below.

\section{Discussion of the results}

\subsection{Sensitivity on the ZAMS model}

We recalled in Sect. 4.2 that our way of building our ZAMS models makes it impossible to maintain a high value of $v / v_{\text {crit }}$ from the ZAMS during the beginning of the MS phase. Activation of the meridional circulation imposes a rapid slowdown of the surface, so the star needs to evolve somewhat in the MS phase in order to again approach the critical limit. At this point, one can ask two questions: 1) would it be possible to build ZAMS models with higher angular momentum content, which would remain near the critical limit throughout the MS phase? 2) How would such behaviour, if possible, change the above results concerning the frequency of stars near the critical limit?

The answer to the first question is yes. It would suffice to distribute the angular momentum or the angular velocity in the ZAMS model differently, for instance, by imposing a nonuniform distribution of $\Omega$ on the ZAMS, allowing more angular momentum to be contained in the inner parts of the star. But this would not change the present results concerning the frequencies of stars near the critical limit. Indeed, these frequencies are computed on the basis of the observed frequencies of stars with given averaged rotational velocities during the MS phase. Stars with higher angular momentum than the most rapid rotators computed here will present higher values of the averaged rotational velocities during the MS and are thus already implicitly accounted for.

\subsection{Sensitivity on the velocity distribution}

Let us first note that the measure of the rotational velocity through the Doppler effect is not free of spurious effects. First this technique only gives access to $v \sin i$ where $i$ is the angle between the line of sight and the rotational axis. This quantity cannot be known except if some other observations as e.g. a rotational period obtained from variability induced by the presence of dark spot at the surface become available. But even in that case, the knowledge of $v$ requires knowing of the radius (more precisely of the equatorial radius). Moreover, if the surface rotates differentially like the solar surface, the period thus obtained might only be partially representative of the surface rotation. Second, due to the von Zeipel theorem, the fast rotating regions of the star, i.e. the zones near the equator, are less luminous than the polar regions, which have low linear velocities. This effect produces an underestimate of the rotational velocities. Townsend et al. (2004) obtain the following behaviour between the FWHM of the HeI 4471 line for a B2-type star and the equatorial velocity. The relation is nearly perfectly linear for $\sin i=1$ and $v / v_{\text {crit }}<0.7$ (for a standard metallicity $9 M_{\odot}$ star, which corresponds to velocities inferior to $300 \mathrm{~km} \mathrm{~s}^{-1}$ on the ZAMS). In that velocity regime, the FWHM measurements, due to the above effects, underestimate the velocities by at most $3-4 \%$.

For $v / v_{\text {crit }}>0.7$, the relation is no longer linear, the FWHM increases much more slowly with the velocity, and it even goes through a maximum, decreasing a little for velocities near the critical limit. In that case, the FWHM becomes a poor indicator of the surface velocity, a star rotating at the critical limit presenting the same line broadening as a star rotating at $80 \%$ of the critical velocity. In other words, this technique may underestimate the real velocity of the star by $20-25 \%$ for very fast rotators (typically for $v / v_{\text {crit }}>0.75$, i.e. for velocities superior to about $340 \mathrm{~km} \mathrm{~s}^{-1}$, for a $9 M_{\odot}$ at standard metallicity). To study this effect, we did the following experiment. We modified the velocity distribution given by Huang \& Gies (2006a) by assuming that between 300 and $420 \mathrm{~km} \mathrm{~s}^{-1}$ all the velocities $v$ should be enhanced by a factor equal to $1+(v-300) / 120 * 0.3$ (i.e. a linear enhancement amounting at most to a factor 1.3 at $420 \mathrm{~km} \mathrm{~s}^{-1}$ ). Using such a modified velocity distribution, we find that the fraction of stars at the critical limit for an age equal to $25 \mathrm{Myr}$ at $Z=0.020$ would be equal to 0.055 instead of 0.049 with the original velocity distribution of Huang \& Gies (2006a, Fig. 20). Thus this effect would not significantly change the results.

To obtain a fraction of stars at the critical limit of about $10 \%$ at $Z=0.020$, it is necessary to considerably increase the fraction of stars born with $\Omega / \Omega_{\text {crit }}$ greater than 0.6 on the ZAMS. Typically passing from the original velocity distribution to a uniform distribution, one obtains that the fraction of stars with values of $\Omega / \Omega_{\text {crit }}$ on the ZAMS superior to $0.6,0.7,0.8$, and 0.9 , passes from $37.5,26.5,15.5$, and $6.5 \%$ to $40,30,20$, and $10 \%$ respectively. Using such a uniform velocity distribution, one finds that the fraction of stars at the critical limit equal $12 \%$ at $Z=0.020$ and age equal to $25 \mathrm{Myr}$ (instead of 5\% with the original velocity distribution). 


\subsection{Sensitivity on mass loss and magnetic fields}

Mass loss, as we have already discussed above, plays a key role in preventing the high mass stars to reach the critical limit. We showed that, by using two different laws for the mass loss rate, very different behaviours are obtained for the $60 M_{\odot}$ models at standard metallicity. It underlines the importance of this ingredient in shaping the evolution of the surface velocity in this mass range. For lower initial mass stars, typically for $20 M_{\odot}$, mass loss becomes lower and lower (at least in the absence of any surface magnetic field).

We can wonder whether mass loss anisotropies would have any impact on the results obtained in this work (which assumed spherical winds). Let us recall that, when a star is rotating at $v / v_{\text {crit }} \geq 0.9$, winds of hot stars will become much more intense at the pole than at the equator. This effect would accelerate the evolution towards the critical limit. However, accounting for this effect would hardly change the evolution we computed here. Indeed, $v / v_{\text {crit }}$ becomes greater than about $0.8-0.9$ only when the surface velocity increases in an already very rapid way (see Fig. 15).

A magnetic field, following the Tayler-Spruit dynamo (Spruit 2002), will tend to impose solid body rotation during the MS phase (Maeder \& Meynet 2005), thus making the approach of the critical limit easier (stronger coupling between the core and the envelope). We can expect that the inclusion of this effect would have an strong impact on the present results, making the fraction of stars at the critical limit greater than in the present work. It has, however, to be noted that at present the Tayler-Spruit dynamo is still very much debated, and some more work is needed before assessing whether this mechanism can be active in stars or not.

\section{Possible links with the Be stars}

There are good indications that the Be star phenomenon is related to the fast rotation of the star. For instance, Martayan et al. (2006a) show that the initial velocities of the Be stars are significantly higher than the initial velocities of the normal B stars, giving some support to the view that only stars with a sufficiently high initial velocity can go through a Be episode. Let us note that, when material is ejected into a Keplerian disk, the star loses angular momentum, slows down, and evolves away from the limit. Secular evolution will then bring the star back towards this limit. Probably the phases in between the loss of material are much longer than the phases during which the star loses material into the Keplerian disk. Thus most of the Be stars might be observed during these quiet phases when they are evolving back towards the limit. It means that the rotation of observed Be stars might be somewhat lower that the rotation at which outbursts occur. If the link between Be stars and fast rotation is now wellaccepted, it is not, however, clear what rotation rate is needed for a star to become a Be star or why this process is mainly restricted to early B type stars. Is rotation the only key parameter governing the transformation of a star into a Be star? When does such a transformation occur? In the present section we would like to provide some answers to these questions.

Comparisons between number fractions of fast rotating stars as predicted by the present models and as observed in clusters (see caption) is shown in Fig. 22. We see first that if we consider only those stars that are strictly at the critical limit (hatched zone I), the prediction falls well below the observed points, all the more so as there are also rapidly rotating stars that are not Be stars, or Be stars that are observed between outbursts. Thus,

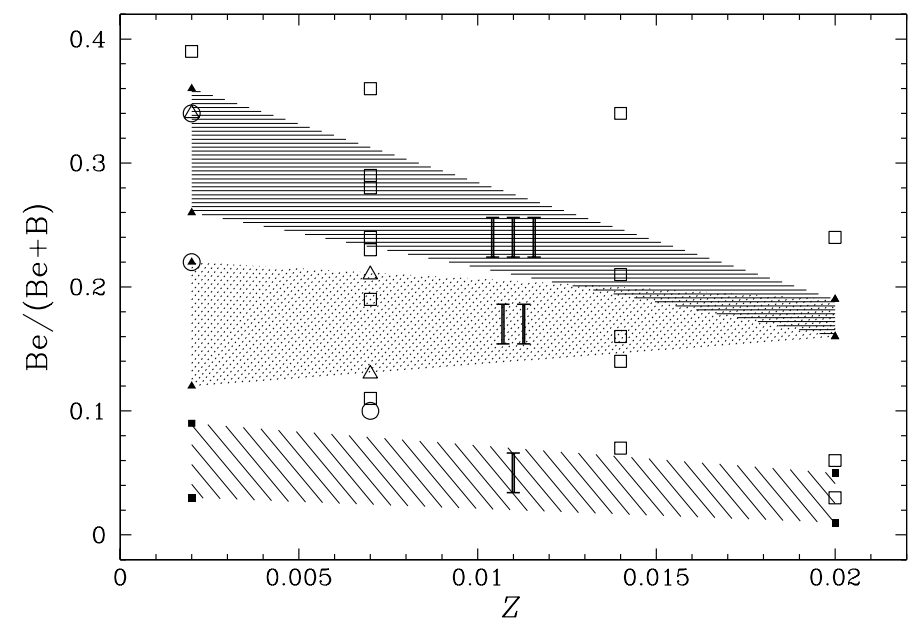

Fig. 22. The empty symbols correspond to the observed fraction of $\mathrm{Be}$ stars among B0-B3 type objects in clusters with ages ranging from 10 to 25 Myr. Data were taken from Wisniewski \& Bjorkman (2006, circles), Keller et al. (1999, triangles) and Maeder et al. (1999, squares). The filled symbols correspond to predictions of the given models for $Z=$ 0.002 and $Z=0.020$ and for ages equal to 10 and $25 \mathrm{Myr}$, respectively (we estimated the fraction of $\mathrm{Be}$ in a two-magnitude interval below the turn off, as did Maeder et al. 1999). The hatched zone labelled by I corresponds to stars predicted at the critical limit. The hatched zone labelled by II shows the fractions of stars with $v / v_{\text {crit }} \geq 0.7$. These two cases were obtained assuming a velocity distribution from Huang \& Gies (2006a). The third hatched region, labelled III, corresponds to stars with $v / v_{\text {crit }} \geq 0.7$ and assumes that the initial distribution of rotation contains faster rotators at low metallicity (see text).

according to the present stellar models, the number fraction of stars at the critical limit is not sufficient to account for the observed fraction of Be stars.

Let us mention that there are two proposed channels for the formation of Be stars: 1) the single star channel, where the critical limit is reached because of the natural evolution of a single rotating star; and 2) the binarity channel, with the spin-up of the member of a binary system through mass transfer from its companion. According to McSwain \& Gies (2005a,b), about $75 \%$ of the detected Be stars may have been spun-up by binary mass transfer, while most of the remaining Be stars were very likely rapid rotators at birth. There are indeed some known binaries among the observed Be stars, but in most cases, no binarity has yet been clearly assessed. It may be very difficult to detect, however, because of the long orbital period and the very small orbital motions. In some cases, decades of careful measurements have been necessary before detecting a companion (see Gies et al. 1998 for $\phi$ Per, Maintz et al. 2005 for 59 Cyg, Rivinius et al. 2004 for FY CMa). According to Negueruela (2007), the binarity channel may be significant, but it is unlikely that most Be stars can form through this channel. From a theoretical point of view, Pols et al. (1991) deduce that probably $40 \%$ (at most $60 \%$ ) of the Be stars may form through mass transfer spin-up. Consequently, the binary channel alone could account for a $(\mathrm{Be} /(\mathrm{B}+\mathrm{Be}))$ ratio of the order of $8-10 \%$ if we take the $(\mathrm{Be} /(\mathrm{B}+\mathrm{Be}))$ value found by Martayan et al. (2006b). In that last case, our prediction thus remains too low even when discounting the binarity channel contribution.

In the frame of the single star scenario, if we assume that the Be phenomenon may occur already at $v / v_{\text {crit }} \geq 0.7$ (see hatched zone II), then the situation improves somewhat; however, the theoretical results do not show any increase in the fraction of Be stars with decreasing metallicity, as some observations indicate 
(Maeder et al. 1999; Wisniewski \& Bjorkman 2006). It might be that part of this trend is due to an observational bias. For instance McSwain \& Gies (2005b) find that Be stars are generally found among the brighter B-stars in their survey of southern sky Galactic clusters. A similar trend was found by Keller et al. (2001) in $\mathrm{h}$ and $\chi$ Per. Since metal-poor clusters are more distant, observations will mainly pick up the brighter end of the B stars sample where the fraction of Be stars is higher. However, it is not clear that such a bias will have a strong impact when clusters of similar ages are compared; provided the observed samples are complete and the count is performed in the same absolute magnitude interval, then the observed number ratios at different metallicities can be compared. To reproduce the observed trend with the metallicity obtained by Maeder et al. (1999) and more recently by Wisniewski \& Bjorkman (2006) with the present models, we have to assume that the fraction of fast rotators at birth is higher in metal-poor regions than in metal-rich one. As a numerical example, supposing that the initial distribution of rotation at $Z=0.002$ is given by a uniform distribution (Fig. 20), we then obtain the hatched zone III, which agrees reasonably well with the observations. From the discussion above, we deduce that to reproduce the observational number fraction of Be stars, we first have to relax the hypothesis that Be stars are strictly at the critical limit: the Be phenomenon should already occur for $v / v_{\text {crit }} \gtrsim 0.7$; second, the distribution of initial values of $\Omega / \Omega_{\text {crit }}$ on the ZAMS at low metallicity should contain a higher fraction of fast rotating stars.

Let us discuss these two conclusions. The first conclusion would tell us that, although fast rotation plays a significant role in transforming the star into a Be star, it is not sufficient alone. Above some value of $v / v_{\text {crit }}$, some other processes, such as pulsations, must help in launching material into a disk.

Now, what about the second conclusion of a higher fraction of fast rotators at low Z? Keller (2004) measured the rotational velocity of $100 \mathrm{MS}$ early B-type stars in clusters of the LMC and compared the results with observations of early B-type stars in clusters of the solar neighbourhood. He finds that the LMC stars are faster rotators than the galactic ones: the mean values of $v \sin i$ is $116 \mathrm{~km} \mathrm{~s}^{-1}$ for the galactic stars and $146 \mathrm{~km} \mathrm{~s}^{-1}$ for the LMC stars. Part of the difference can be due to the difference in mass loss between galactic and LMC stars, but part might also be due to differences in the initial distribution of the velocities. Penny et al. (2004), on the other hand, find no difference between the velocities of O-type stars in the Magellanic Clouds and in the Galaxy. Thus, the picture is at present not clear and probably new surveys, such as the VLT-Flames survey, will provide new insights on that question very soon. At the moment, we still consider the question as open. Present results would, however, clearly favour a higher fraction of stars born with high values of $\Omega / \Omega_{\text {crit }}$ at low metallicities.

A point that the models have to explain is when the Be phenomenon appears during the MS phase. Unfortunately no clear picture emerges from the observations. Some authors find the highest fraction of Be stars among the spectral types B0-B2, appearing most often at ages between 13-25 Myr (Fabregat \& Torrejón 2000). Recently, Frémat et al. (2006), determined the fundamental parameters of the Be stars that would be observable by COROT, found that the main part of their stellar sample falls in the second half of the MS lifetime. This would favour a view according to which the Be star phenomenon tends to appear at the end of the MS phase. Other authors (as e.g. Zorec \& Briot 1997) conclude that the Be phenomenon is not associated with a particular evolutionary phase and can occur during the whole MS phase, at least for some range of initial masses (see
Zorec et al. 2005). With the adopted distribution of initial velocities, present models predict that most near-critical velocity stars will be found at evolved stages during the MS phase and would thus provide an explanation for the observation of Fabregat \& Torrejón (2000). From a physical point of view, we think that the Be star phenomenon may be limited to a given spectral type range for the following reasons. For the earlier spectral types than the favoured one, mass loss prevents the star from approaching the critical limit, and for the later spectral types, the meridional currents are not strong enough to accelerate the envelope. Models would predict a shift towards earlier spectral type when the metallicity decreases due to the decrease in mass loss with the metallicity (extending the range towards earlier spectral type) and the decrease in the meridional velocity in denser layers (increasing the minimum initial mass for meridional currents being efficient enough to accelerate the surface). Of course, beyond these physical reasons are the effects of a possible variation in the distribution of initial rotation as a function of metallicity, which would somewhat blur the above picture. And now, if we relax the condition for becoming a Be star to $v / v_{\text {crit }} \gtrsim 0.7$, we allow this phenomenon to appear earlier in the life of a star.

The present models predict the approach of high values of $v / v_{\text {crit }}(0.8-0.99)$ only when the star is already evolved into the MS phase. As mentioned above, the use of a different internal profile of $\Omega$ in our ZAMS models would allow us to explore ever more extreme cases of rotation, where the star may reach the critical limit, or at least remain near to it, from the beginning of the MS phase. However, these stars should be very rare. The need for the stars to be somewhat evolved to reach high surface velocities implies that these stars should present at their surface $\mathrm{N}$-enrichments. These surface enrichments might, however, be very small for stars in the lower part of the mass range, possibly evolving into a Be star phase (see the cases of our $3 M_{\odot}$ stellar model at $Z=0.020$ in Fig. 17). Observations of $N$ surface enrichments for Be stars are still scarce, probably because of the difficulty of determining abundances from greatly widened absorption lines. Frémat et al. (2004) obtain N/C ratios for four Be stars that are clearly higher than what is observed at the surface of slowly rotating B-type stars. They obtain (N/C)/(N/C) ini of the order of a factor 2, which would correspond closely to what is expected for near critically rotating $9 M_{\odot}$ stars at $Z=0.020$ (see middle panel of Fig. 17).

\section{Conclusions}

From the above computations we can deduce that

1. As long as the mass loss rates are not too high, meridional currents bring the $v / v_{\text {crit }}$ ratio close to 1 during the MS phase, by advecting angular momentum from the inner regions to the outer layers.

2. Since the velocity of the meridional currents in the outer layers scales with the inverse of the density, the process becomes more efficient for stars of higher initial mass and/or higher initial metallicity.

3. At high metallicity, however, mass loss becomes greater and greater and can prevent the stars from reaching the break-up limit.

4. In stellar clusters, one expects that the fraction of stars having $v / v_{\text {crit }} \gtrsim 0.8$ reaches its maximum for ages between 20-32 Myr at $Z=0.020$. This range of ages shifts to older ages at higher metallicities and to younger ages at lower metallicities. Such a shift results from the dependence on the metallicity and the mass of the intensity of the mass loss 
rates and of the velocities of the meridional currents. If we relax the condition and look at stars with $v / v_{\text {crit }} \gtrsim 0.7$, the maximum number will be found in clusters of ages around $10 \mathrm{Myr}$ and below and do so at all non-zero metallicities.

5. Be stars might be the natural outcome of stars with an initial rotational velocity in the upper tail of the initial velocity distribution. To explain their frequency from the rotating stellar models presented here, minimum values of $v / v_{\text {crit }} \gtrsim 0.7$ have to be reached during the MS for the Be star phenomenon to occur.

6. Depending on when this limit is reached, one expects more or less high surface enrichments. If the limit is reached very early during the MS phase, no enrichment is expected, while if this limit is reached at the end of the MS phase, high N/C and N/O ratios are expected. For Be stars developing out of small initial mass stars (typically from $3 M_{\odot}$ at standard metallicity) one expects small surface enrichments or none at all.

7. To reproduce the higher fractions of Be stars at low metallicity, we must assume that, in metal-poor regions, a higher number of stars are born with high values of $\Omega / \Omega_{\text {crit }}$.

In a future work, it will be interesting to explore the effects of a magnetic field. As already mentioned above, this will probably change the results presented here significantly, allowing the stars to approach the critical limit more easily. It will be interesting to see if, in that case, it would still be necessary to invoke a higher proportion of fast rotators at low $Z$.

Acknowledgements. We are very grateful to the referee Douglas R. Gies for his careful reading. His valuable comments have helped us improving the presentation of this paper.

\section{References}

Abt, H. A. 2003, ApJ, 582, 420

Collins, II, G. W. 2004, in Stellar Rotation, ed. A. Maeder, \& P. Eenens, IAU Symp., 215, 3

Cranmer, S. R. 2005, ApJ, 634, 585

de Jager, C., Nieuwenhuijzen, H., \& van der Hucht, K. A. 1988, A\&AS, 72, 259

Decressin, T., Meynet, G., Charbonnel, C., Prantzos, N., \& Ekström, S. 2007, A\&A, 464, 1029

Denissenkov, P. A., Ivanova, N. S., \& Weiss, A. 1999, A\&A, 341, 181

Domiciano de Souza, A., Kervella, P., Jankov, S., et al. 2003, A\&A, 407, L47

Ekström, S., Meynet, G., \& Maeder, A. 2006, in Stellar Evolution at Low Metallicity: Mass Loss, Explosions, Cosmology, ed. H. J. G. L. M. Lamers, N. Langer, T. Nugis, \& K. Annuk, ASP Conf. Ser., 353, 141

Espinosa Lara, F., \& Rieutord, M. 2007, A\&A, 470, 1013

Fabregat, J., \& Torrejón, J. M. 2000, A\&A, 357, 451

Frémat, Y., Zorec, J., Levenhagen, R., et al. 2004, in Stellar Rotation, ed. A. Maeder, \& P. Eenens, IAU Symp., 215, 222

Frémat, Y., Neiner, C., Hubert, A.-M., et al. 2006, A\&A, 451, 1053

Gies, D. R., Bagnuolo, Jr., W. G., Ferrara, E. C., et al. 1998, ApJ, 493, 440

Heger, A., \& Langer, N. 2000, ApJ, 544, 1016

Hirschi, R. 2007, A\&A, 461, 571
Huang, W., \& Gies, D. R. 2006a, ApJ, 648, 580 Huang, W., \& Gies, D. R. 2006b, ApJ, 648, 591 Keller, S. C. 2004, PASA, 21, 310

Keller, S. C., Wood, P. R., \& Bessell, M. S. 1999, A\&AS, 134, 489

Keller, S. C., Grebel, E. K., Miller, G. J., \& Yoss, K. M. 2001, AJ, 122, 248

Kippenhahn, R., \& Thomas, H.-C. 1970, in Stellar Rotation, IAU Coll., 4, 20 Kudritzki, R.-P., \& Puls, J. 2000, ARA\&A, 38, 613

Langer, N. 1997, in Luminous Blue Variables: Massive Stars in Transition, ed. A. Nota, \& H. Lamers, ASP Conf. Ser., 120, 83

Maeder, A., \& Meynet, G. 2000a, A\&A, 361, 159

Maeder, A., \& Meynet, G. 2000b, ARA\&A, 38, 143

Maeder, A., \& Meynet, G. 2001, A\&A, 373, 555

Maeder, A., \& Meynet, G. 2005, A\&A, 440, 1041

Maeder, A., \& Zahn, J.-P. 1998, A\&A, 334, 1000

Maeder, A., Grebel, E. K., \& Mermilliod, J.-C. 1999, A\&A, 346, 459

Maintz, M., Rivinius, T., Stahl, O., Stefl, S., \& Appenzeller, I. 2005, Publications of the Astronomical Institute of the Czechoslovak Academy of Sciences, 93, 21

Martayan, C., Frémat, Y., Hubert, A.-M., et al. 2006a, A\&A, 452, 273

Martayan, C., Hubert, A. M., Floquet, M., et al. 2006b, A\&A, 445, 931

McSwain, M. V., \& Gies, D. R. 2005a, ApJ, 622, 1052

McSwain, M. V., \& Gies, D. R. 2005b, ApJS, 161, 118

Meilland, A., Stee, P., Vannier, M., et al. 2007, A\&A, 464, 59

Meynet, G., \& Maeder, A. 1997, A\&A, 321, 465

Meynet, G., \& Maeder, A. 2000, A\&A, 361, 101

Meynet, G., \& Maeder, A. 2003, A\&A, 404, 975

Meynet, G., \& Maeder, A. 2005, A\&A, 429, 581

Meynet, G., \& Maeder, A. 2007, A\&A, 464, L11

Meynet, G., Mermilliod, J.-C., \& Maeder, A. 1993, A\&AS, 98, 477

Meynet, G., Ekström, S., \& Maeder, A. 2006a, A\&A, 447, 623

Meynet, G., Maeder, A., Hirschi, R., Ekstrom, S., \& Chiappini, C. 2006b, in International Symposium on Nuclear Astrophysics - Nuclei in the Cosmos

Meynet, G., Ekström, S., Maeder, A., \& Barblan, F. 2007, in Active OB-Stars:

Laboratories for Stellare and Circumstellar Physics, ed. A. T. Okazaki, S. P., Owocki, \& S. Stefl, ASP Conf. Ser., 361, 325

Negueruela, I. 2007, in Active OB-Stars: Laboratories for Stellare and Circumstellar Physics, ed. A. T. Okazaki, S. P. Owocki, \& S. Stefl, ASP Conf. Ser., 361,376

Owocki, S. P. 2004, in Stellar Rotation, ed. A. Maeder, \& P. Eenens, IAU Symp., 215,515

Penny, L. R., Sprague, A. J., Seago, G., \& Gies, D. R. 2004, ApJ, 617, 1316

Pols, O. R., Cote, J., Waters, L. B. F. M., \& Heise, J. 1991, A\&A, 241, 419

Porter, J. M., \& Rivinius, T. 2003, PASP, 115, 1153

Rivinius, T., Štefl, S., Maintz, M., Stahl, O., \& Baade, D. 2004, A\&A, 427, 307

Sackmann, I.-J., \& Anand, S. P. S. 1970, ApJ, 162, 105

Schnerr, R. S., Henrichs, H. F., Oudmaijer, R. D., \& Telting, J. H. 2006, A\&A, 459, L21

Spruit, H. C. 2002, A\&A, 381, 923

Talon, S., \& Zahn, J.-P. 1997, A\&A, 317, 749

Townsend, R. H. D., Owocki, S. P., \& Howarth, I. D. 2004, MNRAS, 350, 189

Vink, J. S., de Koter, A., \& Lamers, H. J. G. L. M. 2000, A\&A, 362, 295

von Zeipel, H. 1924, MNRAS, 84, 665

Wisniewski, J. P., \& Bjorkman, K. S. 2006, ApJ, 652, 458

Woosley, S. E. 1993, ApJ, 405, 273

Woosley, S. E., \& Heger, A. 2006, in Gamma-Ray Bursts in the Swift Era, ed. S. S. Holt, N. Gehrels, \& J. A. Nousek, American Institute of Physics Conf. Ser., 836, 398

Yoon, S.-C., \& Langer, N. 2005, A\&A, 443, 643

Zahn, J.-P. 1992, A\&A, 265, 115

Zorec, J., \& Briot, D. 1997, A\&A, 318, 443

Zorec, J., Frémat, Y., \& Cidale, L. 2005, A\&A, 441, 235 